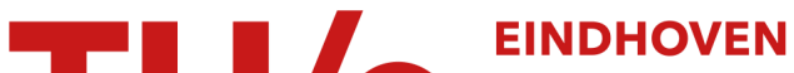 \\ UNIVERSITY OF \\ TECHNOLOGY
}

\section{Output regulation for linear systems subject to input saturation}

\author{
Citation for published version (APA): \\ Lin, Z., Stoorvogel, A. A., \& Saberi, A. (1994). Output regulation for linear systems subject to input saturation. \\ (Memorandum COSOR; Vol. 9402). Technische Universiteit Eindhoven.
}

\section{Document status and date:}

Published: 01/01/1994

\section{Document Version:}

Publisher's PDF, also known as Version of Record (includes final page, issue and volume numbers)

\section{Please check the document version of this publication:}

- A submitted manuscript is the version of the article upon submission and before peer-review. There can be important differences between the submitted version and the official published version of record. People interested in the research are advised to contact the author for the final version of the publication, or visit the $\mathrm{DOI}$ to the publisher's website.

- The final author version and the galley proof are versions of the publication after peer review.

- The final published version features the final layout of the paper including the volume, issue and page numbers.

Link to publication

\section{General rights}

Copyright and moral rights for the publications made accessible in the public portal are retained by the authors and/or other copyright owners and it is a condition of accessing publications that users recognise and abide by the legal requirements associated with these rights.

- Users may download and print one copy of any publication from the public portal for the purpose of private study or research.

- You may not further distribute the material or use it for any profit-making activity or commercial gain

- You may freely distribute the URL identifying the publication in the public portal.

If the publication is distributed under the terms of Article $25 \mathrm{fa}$ of the Dutch Copyright Act, indicated by the "Taverne" license above, please follow below link for the End User Agreement:

www.tue.nl/taverne

Take down policy

If you believe that this document breaches copyright please contact us at:

openaccess@tue.nl

providing details and we will investigate your claim. 
EINDHOVEN UNIVERSITY OF TECHNOLOGY

Department of Mathematics and Computing Science

Memorandum COSOR 94-02

Output regulation for linear systems

subject to input saturation

Z. Lin

A.A. Stoorvogel

A. Saberi

Eindhoven, January 1994

The Netherlands 


\title{
OUTPUT REGULATION FOR LINEAR SYSTEMS SUBJECT TO INPUT SATURATION
}

\author{
Zongli Lin ${ }^{1}$ \\ School of Electrical Engineering \\ and Computer Science \\ Washington State University \\ Pullman, WA 99164-2752 \\ U.S.A.
}

\author{
Anton A. Stoorvogel ${ }^{2}$ \\ Department of Mathematics \\ and Computing Science \\ Eindhoven University of Technology \\ P.O. Box 513, 5600 MB Eindhoven \\ the Netherlands
}

\author{
Ali Saberi \\ School of Electrical Engineering \\ and Computer Science \\ Washington State University \\ Pullman, WA 99164-2752 \\ U.S.A.
}

January 10, 1994

\begin{abstract}
The purpose of this paper is to examine the problem of controlling a linear time-invariant system subject to input saturation in order to have its output track (or reject) a family of reference (or disturbance) signals produced by some external generator. It is shown that a semi-global framework for this problem is a natural one. Within this framework, a set of solvability conditions are given and feedback laws which solve the problem are constructed. The theory developed in this paper incorporates earlier results established for linear systems without input saturation.
\end{abstract}

\footnotetext{
${ }^{1}$ This work is performed when the first author visited Eindhoven University of Technology supported by the Dutch Network on Systems and Control.

${ }^{2}$ The research of dr. A.A. Stoorvogel has been made possible by a fellowship of the Royal Netherlands Academy of Sciences and Arts.
} 


\section{Introduction}

Recently, there has been renewed interest in the study of linear systems subject to input saturation, probably due to a wide recognition of the inherent constraints on the control input. Most of the results in this study pertain to the problem of global and semi-global stabilization. A crucial result on the subject of stabilization of such systems appeared in [14] where it was established that linear systems with saturating input can be globally asymptotically stabilized if and only if the system in the absence of saturation is asymptotically null controllable ${ }^{1}$. Another crucial result related to the stabilization problem is that, in general, linear feedback cannot be used for the purpose of global asymptotic stabilization of linear systems subject to input saturation. This "negative result" was first pointed out in [6] and elaborated on in [15]. A particular nonlinear feedback law using multiple saturation functions for the global asymptotic stabilization of such systems was initiated in [17] and completed in [16]. In response to this "negative result", the semi-global view-point for stabilization of asymptotically null controllable systems with input saturation was proposed in [8], [10], [11] and [9]. In [8], it was shown that one can semi-globally exponentially stabilize a linear asymptotically null controllable system subject to input saturation using linear feedback laws. In other words, the basin of attraction of a linear asymptotically null controllable system subject to input saturation can be made arbitrarily large using appropriately tuned linear feedback laws.

A natural research problem following the stabilization problem is the problem of output regulation (or rejection) of references (or disturbances) generated by some external system, usually called the exosystem. This problem is called the output regulation problem. The only recent work on this subject matter is [18] which deals with the global output regulation problem. In [18], a set of solvability conditions for the global output regulation problem was given. However, as shown in Section 3 of this paper, it turns out that these solvability conditions are satisfied for only a few special cases and in general the global output regulation problem as formulated in [18] does not have a solution. Moreover, in those special cases where the solution does exists, one needs to use, in general, nonlinear feedback laws.

In this paper, we focus on the semi-global output regulation problem for linear asymptotically null controllable systems with input saturation. We will study both the state feedback and the error feedback case. The rationale behind the adoption of a semi-global framework for output regulation problem is two-fold. Firstly, the semi-global framework allows us to use linear feedback laws, which is obviously very appealing; and secondly, the semi-global framework seems to be a natural choice when the global output regulation problem, in general, does not have a solution. We naturally extend the output regulator theory for linear systems without input saturation developed by several authors (e.g. [4] and [22]) to the class of linear asymptotically null controllable systems subject to input saturation. More specifically, we introduce the notion of semi-global output regulation problems. We provide a set of solvability conditions and we show that our solvability conditions, for a fairly general class of systems, are also necessary. We also show that, under certain weak assumptions, we cannot weaken these solvability conditions by using nonlinear feedback laws for semi-global regulator problems. However, by example, we show that there are cases where a nonlinear feedback can do better. A crucial component in the design of the regulators in our paper is the low-gain design technique developed in [8]. For this reason, we briefly review the low-gain theory. We also provide a new algebraic Riccati equation based approach for the low-gain design. For the sake of completeness and to facilitate the comparison, we also briefly review the regulator theory for linear systems without input saturation.

\footnotetext{
${ }^{1} \mathrm{~A}$ linear system is asymptotically null controllable if and only of it is stabilizable and all the poles of the open loop system are in the closed left half plane.
} 
We will mostly use standard notation in this paper. $\mathcal{C}^{n}$ denotes the set of $n$ times continuously differentiable functions. For a vector $q=\left(q_{1}, q_{2}, \ldots q_{k}\right)^{\mathrm{T}}$ we define

$$
|q|_{\infty}:=\max _{i}\left|q_{i}\right|
$$

On the other hand, for a vector-valued function $w$ and $T \geq 0$ we define

$$
\|w\|_{\infty}:=\sup _{t}|w(t)|_{\infty}, \quad\|w\|_{\infty, \mathrm{r}}:=\sup _{t \geq T}|w(t)|_{\infty}
$$

Finally $\|\cdot\|$ denotes the standard Euclidean norm.

\section{Preliminaries}

This section consists of two subsections. In the first subsection, we briefly review the linear multivariable regulator theory, while in the second subsection, we review the low gain theory for linear systems.

\subsection{Review of Linear Regulator Theory}

In this subsection, we briefly review the linear multivariable regulator theory. Consider a multivariable linear system as given below,

$$
\left\{\begin{array}{l}
\dot{x}=A x+B u+P w \\
\dot{w}=S w \\
e=C x+Q w
\end{array}\right.
$$

where the first equation of this system describes a plant, with state $x \in \mathbf{R}^{n}$, and input $u \in \mathbf{R}^{m}$, subject to the effect of a disturbance represented by $P w$. The third equation defines the error $e \in \mathbf{R}^{p}$ between the actual plant output $C x$ and a reference signal $Q w$ which the plant output is required to track. The second equation describes an autonomous system, often called the exosystem, with state $w \in \mathbb{R}^{s}$. The exosystem models the class of disturbance or reference signals taken into consideration.

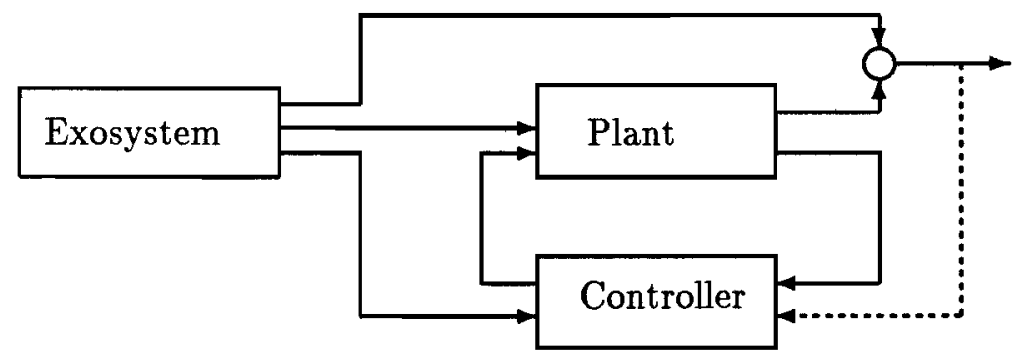

Figure 2.1: Configuration of a linear regulator

The control action to the plant, $u$, can be provided either by state feedback or by error feedback. A state feedback controller has the form

$$
u=F x+G w
$$


Composing (2.1) and (2.2) yields a closed-loop system

$$
\left\{\begin{array}{l}
\dot{x}=(A+B F) x+(P+B G) w, \quad z \in \mathbf{R}^{l} \\
\dot{w}=S w \\
e=C x+Q w
\end{array}\right.
$$

An error feedback controller has the form

$$
\left\{\begin{array}{l}
\dot{z}=A_{c} z+B_{c} e \\
u=C_{c} z+D_{c} e
\end{array}\right.
$$

The interconnection of (2.1) and (2.4) yields a closed-loop system

$$
\left\{\begin{array}{l}
\dot{x}=A x+B C_{c} z+B D_{c}(C x+Q w)+P w \\
\dot{z}=A_{c} z+B_{c}(C x+Q w) \\
\dot{w}=S w \\
e=C x+Q w
\end{array}\right.
$$

The purpose of the control action is to achieve internal stability and output regulation. Internal stability means that, when the exosystem is disconnected (i.e., when $w$ is set equal to 0 ), the closedloop (2.3) [respectively, (2.5)] is asymptotically stable. Output regulation means that for the closed-loop system (2.3) [respectively, (2.5)] and for all initial conditions $(x(0), w(0))$ [respectively, $(x(0), z(0), w(0))]$, we have $e(t) \rightarrow 0$ as $t \rightarrow \infty$. Formally, all of this can be summarized in the following two synthesis problems.

Definition 2.1: (State Feedback Regulator Problem for Linear Systems) Find, if possible, a state feedback law of the form (2.2) such that

1. The system $\dot{x}=(A+B F) x$ is asymptotically stable;

2. For all $(x(0), w(0)) \in \mathbf{R}^{n+s}$, the solution of (2.3) satisfies $\lim _{t \rightarrow \infty} e(t)=0$.

Definition 2.2: (Error Feedback Regulator Problem for Linear Systems) Find, if possible, an error feedback law of the form (2.4) such that

1. The system

$$
\left\{\begin{array}{l}
\dot{x}=A x+B C_{c} z+B D_{c} C x \\
\dot{z}=A_{c} z+B_{c} C x
\end{array}\right.
$$

is asymptotically stable;

2. For all $(x(0), z(0), w(0)) \in \mathbf{R}^{n+l+s}$, the solution of (2.5) satisfies $\lim _{t \rightarrow \infty} e(t)=0$. 
The solution of these two problems (see [4]) is based on the following three assumptions:

A1. The eigenvalues of $S$ have nonnegative real parts.

A2. The pair $(A, B)$ is stabilizable.

A3. The pair $\left(\left[\begin{array}{ll}C & Q\end{array}\right],\left[\begin{array}{cc}A & P \\ 0 & S\end{array}\right]\right)$ is detectable.

The first one of these assumptions does not involve a loss of generality because asymptotically stable modes in the exosystem do not affect the regulation of the output. The second one is indeed necessary for asymptotic stabilization of the closed loop via either state or error feedback. The third one is stronger than the assumption of detectability of the pair $(C, A)$, that would be necessary for the asymptotic stabilization of the closed loop via error feedback, but again does not involve loss of generality, as discussed in detail by Francis in [4]. In fact, if the pair $(C, A)$ is detectable and $A 3$ does not hold, it is always possible to reduce the dimension of the exosystem which actually affects the error, and have - on the reduced system thus obtained - condition A3 satisfied.

The following results, due to Francis, describe necessary and sufficient conditions for the existence of solutions to the above two problems.

Proposition 2.3: Suppose assumptions $A 1$ and $A 2$ hold. Then, the linear state feedback regulator problem is solvable if and only if there exist matrices $\Pi$ and $\Gamma$ which solve the linear matrix equations

$$
\left\{\begin{array}{l}
\mathrm{II} S=A \Pi+B \Gamma+P \\
C \Pi+Q=0
\end{array}\right.
$$

Moreover, a suitable state feedback is given by:

$$
u=-F x+(F \Pi+\Gamma) w
$$

where $F$ is such that $A-B F$ is Hurwitz.

Proposition 2.4: Suppose assumptions A1, A2 and A3 hold. Then, the linear error feedback regulator problem is solvable if and only if there exist matrices $\Pi$ and $\Gamma$ which solve the linear matrix equations (2.6).

Moreover, a suitable error feedback is given by:

$$
\left\{\begin{array}{l}
{\left[\begin{array}{c}
\dot{\hat{x}} \\
\dot{\hat{w}}
\end{array}\right]=\left[\begin{array}{cc}
A & P \\
0 & S
\end{array}\right]\left[\begin{array}{c}
\hat{x} \\
\hat{w}
\end{array}\right]+\left[\begin{array}{c}
B \\
0
\end{array}\right] u+\left[\begin{array}{c}
L_{A} \\
L_{S}
\end{array}\right]\left(e-\left[\begin{array}{ll}
C & Q
\end{array}\right]\left[\begin{array}{c}
\hat{x} \\
\hat{w}
\end{array}\right]\right)} \\
u=-F \hat{x}+(F \Pi+\Gamma) \hat{w}
\end{array}\right.
$$

where $F$ is such that $A-B F$ is Hurwitz and $L_{A}$ and $L_{S}$ are such that the matrix

$$
\bar{A}:=\left[\begin{array}{cc}
A-L_{A} C & P-L_{A} Q \\
-L_{S} C & S-L_{S} Q
\end{array}\right]
$$

is Hurwitz. 
In summary, if assumptions $\mathrm{A} 1, \mathrm{~A} 2$ and $\mathrm{A} 3$ hold, then, the linear error feedback regulator problem is solvable if and only if the state feedback regulator problem is solvable, and the conditions for the existence of solutions can be expressed in terms of the solvability of certain linear matrix equations.

In [7], Hautus has proven that the possibility of solving these matrix equations can be characterized in terms of a comparison between the transmission polynomials of the system (2.1) (in which $u$ is considered as the input and $e$ as the output) and those of the system

$$
\left\{\begin{array}{l}
\dot{x}=A x+B u \\
\dot{w}=S w \\
e=C x
\end{array}\right.
$$

The later can be interpreted as the system obtained from (2.1) by cutting the connections between the exosystem and the plant. More specifically, Hautus proved the following result.

Proposition 2.5: The linear matrix equations (2.6) are solvable if and only if the system (2.1) and (2.9) have the same transmission polynomials.

\subsection{Review of Low-Gain Design for Linear Systems}

In this subsection, we recall a low-gain state feedback design algorithm from [8]. Such a design algorithm yields a family of state feedback gains, parameterized in $\varepsilon$, which are instrumental in proving our main results on semi-global output regulation. We first explicitly construct this family of low-gain state gains and then recall its property in a theorem following the construction. An alternative new approach of obtaining such a low-gain design by solving algebraic Riccati equations will also be given.

\section{Explicit Construction}

Consider the linear system

$$
\dot{x}=A x+B u
$$

where the state $x \in \mathbb{R}^{n}$ and the input $u \in \mathbb{R}^{m}$. Assume that $(A, B)$ is stabilizable and all the eigenvalues of $A$ are located in the closed left half plane. We have the following low-gain state feedback design algorithm.

Step 1 : Find the state transformation $T$ ( [3]) such that $\left(T^{-1} A T, T^{-1} B\right)$ is in the following form

$$
T^{-1} A T=\left[\begin{array}{ccccc}
A_{1} & A_{12} & \cdots & A_{1 p} & A_{1 \bar{c}} \\
0 & A_{2} & \cdots & A_{2 p} & A_{2 \bar{c}} \\
\vdots & \vdots & \ddots & \vdots & \vdots \\
0 & 0 & \cdots & A_{p} & A_{p \bar{c}} \\
0 & 0 & 0 & 0 & A_{\bar{c}}
\end{array}\right], T^{-1} B=\left[\begin{array}{ccccc}
B_{1} & 0 & \cdots & 0 & * \\
0 & B_{2} & \cdots & 0 & * \\
\vdots & \vdots & \ddots & \vdots & \vdots \\
0 & 0 & \cdots & B_{p} & * \\
0 & 0 & 0 & 0 & 0
\end{array}\right]
$$

Here *'s represent submatrices of less interest and for $i=1,2, \cdots, p$,

$$
A_{i}=\left[\begin{array}{ccccc}
0 & 1 & 0 & \cdots & 0 \\
0 & 0 & 1 & \cdots & 0 \\
\vdots & \vdots & \vdots & \ddots & \vdots \\
0 & 0 & 0 & \cdots & 1 \\
-a_{n_{i}}^{i} & -a_{n_{i}-1}^{i} & -a_{n_{i}-2}^{i} & \cdots & -a_{1}^{i}
\end{array}\right], B_{i}=\left[\begin{array}{c}
0 \\
0 \\
\vdots \\
0 \\
1
\end{array}\right]
$$


Furthermore, $\left(A_{i}, B_{i}\right)$ is controllable, all the eigenvalues of $A_{i}$ are in the closed left half $s$-plane, and all the eigenvalues of $A_{\bar{c}}$ are in the open left half plane.

Step 2 : For each $\left(A_{i}, B_{i}\right)$, let $F_{i}(\varepsilon) \in \mathbf{R}^{1 \times n_{i}}$ be the state feedback gain such that

$$
\lambda\left(A_{i}-B_{i} F_{i}(\varepsilon)\right)=-\varepsilon+\lambda\left(A_{i}\right) \in \mathbb{C}^{-}
$$

Note that $F_{i}(\varepsilon)$ is unique.

Step 3 : Form a state feedback gain matrix $F(\varepsilon)$ as

$$
F(\varepsilon)=\left[\begin{array}{cccccc}
F_{1}\left(\varepsilon^{\left(r_{2}+1\right)\left(r_{3}+1\right) \cdots\left(r_{p}+1\right)}\right) & 0 & \cdots & 0 & 0 & 0 \\
0 & F_{2}\left(\varepsilon^{\left(r_{3}+1\right)\left(r_{1}+1\right) \cdots\left(r_{p}+1\right)}\right) & \cdots & 0 & 0 & 0 \\
\vdots & \vdots & \ddots & \vdots & \vdots & \vdots \\
0 & 0 & \cdots & F_{p-1}\left(\varepsilon^{r_{p}+1}\right) & 0 & 0 \\
0 & 0 & \cdots & 0 & F_{p}(\varepsilon) & 0 \\
0 & 0 & \cdots & 0 & 0 & 0
\end{array}\right] T^{-1}
$$

where $r_{i}$ is the largest algebraic multiplicity of the eigenvalues of $A_{i}$.

With this choice of state feedback gain $F(\varepsilon)$, we have the following theorem.

Theorem 2.6: Consider the linear system (2.10). Assume that the pair $(A, B)$ is stabilizable and all the eigenvalues of $A$ are located in the closed left half $s$-plane. Then, for the state feedback gain given by (2.11), the closed-loop system

$$
\dot{x}=(A-B F(\varepsilon)) x
$$

is asymptotically stable for all $\varepsilon>0$. Moreover, there exists an $\varepsilon^{*}>0$ such that for all $\varepsilon \in\left(0, \varepsilon^{*}\right]$,

$$
\begin{aligned}
& \|F(\varepsilon)\| \leq \alpha \varepsilon \\
& \left\|F(\varepsilon) e^{(A-B F(\varepsilon)) t}\right\| \leq \beta \varepsilon e^{-\varepsilon^{\gamma} t}
\end{aligned}
$$

where $\alpha$ and $\beta$ are positive constants independent of $\varepsilon$ and $\gamma$ is a positive integer also independent of $\varepsilon$.

Proof: See [8].

An alternative approach to the design of low-gain state feedback is based on the solutions of the algebraic Riccati equation (ARE). Here again we start with the construction of the family of state feedback gains using ARE-based method. The property of this family of state feedback gains is given in the theorem following the construction.

\section{ARE-Based Approach}

Consider the linear system

$$
\dot{x}=A x+B u
$$

where the state $x \in \mathbf{R}^{n}$ and the input $u \in \mathbf{R}^{m}$. Assume that $(A, B)$ is stabilizable and all the eigenvalues of $A$ are located in the closed left half plane. We have the following ARE-based lowgain state feedback design algorithm. 
Step 1 : Consider the linear quadratic control problem with a cost function given by

$$
\mathcal{J}\left(x_{0}, u\right):=\int_{0}^{\infty} u^{\mathrm{T}}(t) u(t)+\varepsilon x^{\mathrm{T}}(t) x(t) d t
$$

Step 2 : Solve the following algebraic Riccati equation

$$
A^{\mathrm{T}} P(\varepsilon)+P(\varepsilon) A-P(\varepsilon) B B^{\mathrm{T}} P(\varepsilon)+\varepsilon I=0
$$

to find a unique positive definite solution $P(\varepsilon)$ and obtain the state feedback gain

$$
F(\varepsilon)=B^{\mathrm{T}} P(\varepsilon)
$$

where the existence of the unique positive definite solution of the algebraic Riccati equation is established in the following lemma.

Lemma 2.7: Assume $(A, B)$ is stabilizable and $A$ has all its eigenvalues in the closed left-half plane. Then, for all $\varepsilon>0$ there exists a unique matrix $P(\varepsilon)>0$ which solves the algebraic Riccati equation (2.17). Moreover, $P(\varepsilon) \rightarrow 0$ as $\varepsilon \rightarrow 0$. Finally, the state feedback $u=-B^{\mathrm{T}} P(\varepsilon) x$ stabilizes the system and is such that $\inf _{u} \mathcal{J}\left(x_{0}, u\right) \rightarrow 0$ for all $x_{0} \in \mathbf{R}^{n}$ as $\varepsilon \rightarrow 0$.

Proof : The existence of a unique positive definite solution $P(\varepsilon)$ for all $\varepsilon>0$ has been established in [20]. Moreover, $A-B B^{\mathrm{T}} P(\varepsilon)$ is asymptotically stable for all $\varepsilon>0$. The same paper established that for $\varepsilon=0$ there is a unique solution $P(0)=0$ for which $A-B B^{\mathrm{T}} P(0)$ has all eigenvalues in the closed left half plane. Continuity of the solution of the algebraic Riccati equation for $\varepsilon=0$ (in other words that $P(\varepsilon) \rightarrow P(0)=0$ as $\varepsilon \rightarrow 0$ ) has been established in [19]. The final result follows from the fact that $\inf _{u} \mathcal{J}\left(x_{0}, u\right)=x_{0}^{\mathrm{T}} P(\varepsilon) x_{0}$ (see $[20]$ ).

Remark : We would like to note that, unlike the explicit construction of the state feedback gain, this ARE-based approach of obtaining the state feedback gain matrix $F(\varepsilon)$ requires the solution of a parameterized algebraic Riccati equation, which can become very stiff numerically, although it is conceptually appealing.

With the state feedback law (2.18), the closed-loop system has the following property.

Theorem 2.8: Assume that $(A, B)$ is stabilizable and $A$ has all its eigenvalues in the closed left-half plane. Then, for the state feedback gain as given by (2.18), the closed-loop system

$$
\dot{x}=(A-B F(\varepsilon)) x
$$

is asymptotically stable for all $\varepsilon>0$ and for all $\varepsilon \in(0,1]$,

$$
\begin{aligned}
& \|F(\varepsilon)\| \leq \alpha(\varepsilon) \\
& \left\|F(\varepsilon) e^{(A-B F(\varepsilon)) t}\right\| \leq \beta(\varepsilon) e^{-\varepsilon \gamma t}
\end{aligned}
$$

for some $\alpha(\varepsilon)>0, \beta(\varepsilon)>0$ and $\gamma>0$. Moreover, $\alpha(\varepsilon), \beta(\varepsilon) \rightarrow 0$ as $\varepsilon \rightarrow 0$. 
Proof: (2.20) follows trivially from Lemma 2.7. We remain to show (2.21). Using the algebraic Riccati equation (2.17), we find that, for $\varepsilon \in(0,1]$,

$$
\frac{d}{d t} x^{\mathrm{T}}(t) P(\varepsilon) x(t)=-\left\|B^{\mathrm{T}} P(\varepsilon) x\right\|^{2}-\varepsilon\|x\|^{2} \leq-2 \varepsilon \gamma\left\|P^{1 / 2}(\varepsilon) x\right\|^{2}
$$

for some $\gamma>0$. Hence

$$
\left\|P^{1 / 2}(\varepsilon) x(t)\right\| \leq e^{-\varepsilon \gamma t}\left\|P^{1 / 2}(\varepsilon) x(0)\right\|
$$

Finally,

$$
\left\|F(\varepsilon) e^{(A-B F(\varepsilon)) t} x(0)\right\|=\left\|B^{\mathrm{T}} P(\varepsilon) x(t)\right\| \leq\|B\|\left\|P^{1 / 2}(\varepsilon)\right\|^{2} e^{-\varepsilon \gamma t}\|x(0)\|
$$

Since (2.24) is true for all $x(0) \in \mathbf{R}^{n}$, it follows trivially that

$$
\left\|F(\varepsilon) e^{(A-B F(\varepsilon)) t}\right\| \leq\|B\|\left\|P^{1 / 2}(\varepsilon)\right\|^{2} e^{-\varepsilon \gamma t}
$$

The proof is then completed by taking $\beta(\varepsilon)=\|B\|\left\|P^{1 / 2}(\varepsilon)\right\|^{2} \rightarrow 0$ as $\varepsilon \rightarrow 0$.

\section{Global Regulator Problem for Linear Systems Subject to Input Saturation}

In this section, we consider the regulator problem when the plant inputs are subject to saturation. More specifically, we consider a multivariable system with inputs that are subject to saturation together with an exosystem that generates disturbance and reference signals as described by the following system

$$
\left\{\begin{array}{l}
\dot{x}=A x+B \sigma(u)+P w \\
\dot{w}=S w \\
e=C x+Q w
\end{array}\right.
$$

where $x \in \mathbf{R}^{n}, w \in \mathbf{R}^{s}, u \in \mathbf{R}^{m}, e \in \mathbf{R}^{p}$, and $\sigma$ is a vector-valued saturation function defined as

$$
\sigma(s)=\left[\sigma_{1}\left(s_{1}\right), \sigma_{1}\left(s_{2}\right), \cdots, \sigma_{1}\left(s_{m}\right)\right]^{\prime}
$$

with

$$
\sigma_{1}(s)=\left\{\begin{array}{cc}
s_{i} & \text { if }\left|s_{i}\right| \leq 1 \\
-1 & \text { if } s_{i}<-1 \\
1 & \text { if } s_{i}>1
\end{array}\right.
$$

Because of the presence of the saturation function $\sigma$, the system (3.3) is nonlinear. Note that we can also treat different saturation levels, even differences between channels, by simple scaling. The global regulator problem for such a nonlinear system was first formulated by Teel in [18]. More specifically, Teel formulated the global state feedback regulator problem and the global error feedback regulator problem as follows.

Definition 3.1: (Global State Feedback Regulator Problem for Linear Systems Subject to Input Saturation) Find, if possible, a feedback $u=\alpha(x, w)$ such that 
1. The equilibrium $x=0$ of

$$
\dot{x}=A x+B \sigma(\alpha(x, 0))
$$

is globally asymptotically stable and locally exponentially stable;

2. For all $(x(0), w(0)) \in \mathbf{R}^{n+s}$, the solution of the closed-loop system satisfies

$$
\lim _{t \rightarrow \infty} e(t)=0
$$

Definition 3.2: (Global Error Feedback Regulator Problem for Linear Systems Subject to Input Saturation) Find, if possible, a dynamic error feedback $u=\theta(z), \dot{z}=\eta(z, e)$ where $z \in \mathbf{R}^{l}$ such that

1. The equilibrium $(x, z)=(0,0)$ of

$$
\left\{\begin{array}{l}
\dot{x}=A x+B \sigma(\theta(z)) \\
\dot{z}=\eta(z, C x)
\end{array}\right.
$$

is globally asymptotically stable and locally exponentially stable.

2. For all $(x(0), z(0), w(0)) \in \mathbf{R}^{n+l+s}$, the solution of the closed-loop system satisfies

$$
\lim _{t \rightarrow \infty} e(t)=0 \text {. }
$$

A set of sufficient conditions for the above global regulator problems to have a solution are given in [18].

The global regulation as defined in the above is clearly a very desirable property. Unfortunately it turns out that only in very special circumstances can we achieve global regulation. In fact, the global error feedback regulator problem as formulated in Definition 3.2 basically has no solution. This is established in the following lemma.

Lemma 3.3: Suppose assumptions $A 1, A 2$ and $A 3$ hold. Then there exist initial conditions $w_{0}$ for $w$ such that there exists no input $u$ or initial condition $x(0)$ for which the closed-loop system satisfies $\lim _{t \rightarrow \infty} e(t)=0$.

Proof : We study the system (3.1) without the saturation element, i.e. we have the following system:

$$
\left\{\begin{array}{l}
\dot{x}=A x+B v+P w \\
\dot{w}=S w \\
e=C x+Q w
\end{array}\right.
$$

Let $w_{0}$ with $\left\|w_{0}\right\|=1$ be an eigenvector of $S$ belonging to an eigenvalue $\lambda$ of $S$.

Suppose $\lambda$ is an eigenvalue with strictly positive real part. We can decompose $e$ in three components. One due to the possibly non-zero initial condition $x_{0}$, one due to the bounded input $v$ and one due to the initial condition $w_{0}$. The first two can only grow polynomially in time since all eigenvalues of $A$ are in the closed left half plane. On the other hand the effect of $w_{0}$ will, due to 
detectability assumption A3, grow exponentially in time. Therefore $e$ will also grow exponentially in time and we cannot achieve regulation.

If $\lambda$ does not have a strictly positive real part then, by assumption $A 1$, it must lie on the imaginary axis. In that case, $w$ will be periodic and bounded since $w(t)=e^{\lambda t} w_{0}$.

We define the minimal amplitude of an input signal which achieves tracking and minimize this over all possible initial conditions of the plant:

$$
\mathcal{J}\left(w_{0}\right):=\inf _{v, x_{0}}\left\{\|v\|_{\infty} \mid v \text { is such that } \lim _{t \rightarrow \infty} e(t)=0 \text { where } x(0)=x_{0} \text { and } w(0)=w_{0}\right\}
$$

Suppose $\mathcal{J}\left(w_{0}\right)=0$. We take a minimizing sequence $\left\{v_{i}, x_{0, i}\right\}$ for the above optimization problem. For each $v_{i}$ there exists a $T_{i}$ such that $\left\|e\left(T_{i}+t\right)\right\|<1 / i$ for all $t>0$ and $w\left(T_{i}\right)=w_{0}$ (use that $w$ is periodic). Define

$$
\bar{v}_{i}(t):=v_{i}\left(T_{i}+t\right)
$$

Then $\left\|\bar{v}_{i}\right\|_{\infty} \leq\left\|v_{i}\right\|_{\infty} \rightarrow 0$ as $i \rightarrow \infty$. The output $\bar{e}_{i}$ resulting from input $\bar{v}_{i}$ and initial conditions

$$
\bar{x}(0)=\bar{x}_{0, i}:=x\left(T_{i}\right)
$$

and $\bar{w}(0)=w_{0}$ satisfies $\left\|\bar{e}_{i}\right\|_{\infty}<1 / i$. The latter is straightforward since $\bar{e}_{i}(t)=e\left(T_{i}+t\right)$.

Pick any $T>0$. On $[0, T]$ the input $\bar{v}_{i}$ converges in $L_{\infty}$ norm to 0 . Similarly $\bar{e}_{i}$ converges to 0 uniformly on $[0, T]$. Define $f: \mathbb{R}^{n} \rightarrow L_{\infty}[0, T]$ by $[f(z)](t)=C e^{A t} z$. We can check that $g \in L_{\infty}[0, T]$ is in the closure of the image of $f$ where

$$
g(t):=\int_{0}^{t} C e^{A(t-\tau)} P w(\tau) d \tau+Q w(t)
$$

Since $f$ is a finite rank operator we know the image is closed and hence $g$ is in the image of $f$, i.e. there exists an $\tilde{x}_{0}$ such that $f\left(\tilde{x}_{0}\right)=-g$. We find:

$$
\left(\begin{array}{ll}
C & Q
\end{array}\right) \exp \left[\left(\begin{array}{cc}
A & P \\
0 & S
\end{array}\right) t\right]\left(\begin{array}{c}
\tilde{x}_{0} \\
w_{0}
\end{array}\right)=0
$$

for $t \in[0, T]$. This immediately implies that (3.5) holds for all $t$. On the other hand $w(t) \nrightarrow 0$ as $t \rightarrow \infty$. However this contradicts the detectability assumption A3. Therefore we have $\mathcal{J}\left(w_{0}\right)>0$.

Hence we find that for $w(0)=2 w_{0} / \mathcal{J}\left(w_{0}\right)$ any input $v$ which achieves regulation satisfies $\|v\|_{\infty} \geq 2$. Therefore $v=\sigma(u)$ will never be able to achieve regulation, i.e. no input $u$ to (3.1) exists for which $e(t) \rightarrow 0$ as $t \rightarrow \infty$.

The above involves complex inputs. By working with the real or complex part of the signals we can avoid this technical problem.

Remark : Assumptions $A 2$ and $A 3$ as well as the requirement that all eigenvalues of $A$ are in the closed left half plane are clearly necessary for the solvability of the regulator problem using error feedback for a system with input saturation. Only assumption A1 is not strictly necessary. However, using the above lemma it is easy to derive that the global regulator problem with error feedback is solvable only if assumptions $A 2$ and $A 3$ are satisfied, $S$ is asymptotically stable, and all eigenvalues of $A$ are in the closed left half plane. This means that $w$ converges to zero and any globally stabilizing controller for the plant will solve the regulator problem. Hence the global error feedback regulator problem basically has no solution. 
Remark : There are cases where the global state feedback regulator problem is solvable. However, it is necessary that the set of linear equations (4.11) is solvable. Since there are more equations than unknowns it is obvious that the state feedback regulator problem is solvable only in a few special cases. Furthermore, these special cases are generally solvable only using nonlinear feedback regulators.

The above clearly yields a good argument to restrict attention to initial conditions $w(0)$ inside a given compact set. However, in the theory of stabilization of linear systems subject to saturation, the step from global initial conditions to initial conditions inside a compact set has already been made. This has been named semi-global stabilization. Since, in most cases, we have to restrict attention to initial conditions for $w$ inside a compact set anyway this yields good motivation to direct our attention to a semi-global setting. Of course this also yields the well-known advantage that we can achieve regulation with linear compensators. The semi-global setting will be the main topic of this paper.

\section{Semi-Global Regulator Problem for Linear Systems Subject to Input Satura- tion}

We split this section into two parts. In the first part we study the semi-global linear state feedback regulator problem where all signals are available for feedback and it suffices to look at static feedbacks. In the second part we study the semi-global error feedback regulator problem where only the error signal is available for feedback and we have to resort to dynamic feedback.

\subsection{Static State Feedback}

The semi-global linear state feedback regulator problem can be formulated as follows.

Definition 4.1: (Semi-Global Linear State Feedback Regulator Problem) Consider the system (3.1) and a compact set $\mathcal{W}_{0} \subset \mathbf{R}^{s}$. The semi-global linear state feedback regulator problem is defined as follows.

For any a priori given (arbitrarily large) bounded set $\mathcal{X}_{0} \subset \mathbf{R}^{n}$, find, if possible, a linear static feedback law $u=F x+G w$ such that

1. The equilibrium $x=0$ of

$$
\dot{x}=A x+B \sigma(F x)
$$

is locally exponentially stable with $\mathcal{X}_{0}$ contained in its basin of attraction;

2. For all $x(0) \in \mathcal{X}_{0}$ and $w(0) \in \mathcal{W}_{0}$, the solution of the closed-loop system satisfies

$$
\lim _{t \rightarrow \infty} e(t)=0
$$

Remark: We would like to emphasize that our definition of semi-global linear state feedback regulator problem does not view the set of initial conditions of the plant as given data. The set of given data consists of the models of the plant and the exosystem and the set of initial conditions for the exosystem. Therefore, any solvability conditions we obtain must be independent of the set of initial conditions of the plant, $\mathcal{X}_{0}$. 
The solvability conditions for semi-global linear state feedback regulator problem are given in the following theorem.

Theorem 4.2: Consider the system (3.1) and the given compact set $\mathcal{W}_{0} \subset \mathbf{R}^{s}$. The semi-global linear state feedback regulator problem is solvable if

1. $(A, B)$ is stabilizable and $A$ has all eigenvalues in the closed left half plane;

2. There exist matrices $\Pi$ and $\Gamma$ such that:

(a) They solve the following linear matrix equations :

$$
\left\{\begin{array}{l}
\Pi S=A \Pi+B \Gamma+P \\
C \Pi+Q=0
\end{array}\right.
$$

(b) There exist a $\delta>0$ and a $T \geq 0$ such that $\|\Gamma w\|_{\infty, \mathrm{T}} \leq 1-\delta$ for all $w$ with $w(0) \in \mathcal{W}_{0}$.

Proof: We prove this theorem by first explicitly constructing a family of linear static state feedback laws, parameterized in $\varepsilon$, and then showing that for each given set $\mathcal{X}_{0}$, there exists an $\varepsilon^{*}>0$ such that for all $\varepsilon \in\left(0, \varepsilon^{*}\right]$, both items 1 and 2 of Definition 4.1 hold. The family of linear static state feedback laws we construct takes the following form

$$
u=-F(\varepsilon) x+(F(\varepsilon) \Pi+\Gamma) w
$$

where $F(\varepsilon)$ is a state feedback gain matrix, parameterized in $\varepsilon$ and chosen in such a way that $A-B F(\varepsilon)$ is Hurwitz for all $\varepsilon>0$ and

$$
\left\|F(\varepsilon) e^{(A-B F(\varepsilon)) t}\right\|_{\infty} \leq \beta(\varepsilon),
$$

where $\beta$ is a positive-valued function satisfying $\lim _{\varepsilon \rightarrow 0} \beta(\varepsilon)=0$. The existence and the explicit construction of such a gain matrix $F(\varepsilon)$ is established in Section 2.2.

With this family of feedback laws, the system (4.1) is written as

$$
\dot{x}=A x+B \sigma(-F(\varepsilon) x)
$$

To show that item 1 of Definition 4.1 holds, let us consider the system (4.6) in the absence of the saturation elements. The system then takes the form

$$
\dot{x}=(A-B F(\varepsilon)) x
$$

It then follows from (4.5) that there exists an $\varepsilon_{1}^{*}>0$ such that for all $\varepsilon \in\left(0, \varepsilon_{1}^{*}\right]$ we have:

$$
\|F(\varepsilon) x\|_{\infty} \leq 1, \quad \forall x(0) \in \mathcal{X}_{0}
$$

This shows that for all $\varepsilon \in\left(0, \varepsilon_{1}^{*}\right]$ and for all $x(0) \in \mathcal{X}_{0}$, the system (4.6) operates in the linear regions of the saturation elements and hence we conclude that the equilibrium $x=0$ of the system (4.6) is locally exponentially stable with $\mathcal{X}_{0}$ contained in its basin of attraction.

To show the rest of the theorem, let us introduce an invertible, triangular coordinate change $\xi=x-\Pi w$. Using Condition 2(a), we have

$$
\begin{aligned}
\dot{\xi} & =\dot{x}-\Pi \dot{w} \\
& =A x+B \sigma(u)+P w-\Pi S w \\
& =A \xi+B(\sigma(u)-\Gamma w)
\end{aligned}
$$


With the family of state feedback laws given above, the closed-loop system can be written as

$$
\dot{\xi}=A \xi+B[\sigma(\Gamma w-F(\varepsilon) \xi)-\Gamma w]
$$

By Condition 2(b), $\|\Gamma w\|_{\infty, \mathrm{T}}<1-\delta$. Moreover, $\xi(T)$ belongs to a bounded set independent of $\varepsilon$ since $\xi(0)$ is bounded and $\xi(T)$ is determined by a linear differential equation with bounded inputs $\sigma(u)$ and $\Gamma w$. If we consider the system (4.9), from time $T$ onwards, without saturation element, we obtain

$$
\dot{\xi}=(A-B F(\varepsilon)) \xi
$$

Since $\xi(T)$ is bounded, (4.5) and (4.10) imply that there exists $\varepsilon_{2}^{*}>0$ such that, for all $\varepsilon \in\left(0, \varepsilon_{2}^{*}\right]$,

$$
\|F(\varepsilon) \xi\|_{\infty, \mathrm{T}} \leq \delta
$$

We can conclude that the system will operate within the linear region of the saturation elements for all $t \geq T$ if $\varepsilon \in\left(0, \varepsilon_{2}^{*}\right]$ and due to stability of $A-B F(\varepsilon)$ we find $e(t)=C \xi(t) \rightarrow 0$ as $t \rightarrow \infty$.

Finally, taking $\varepsilon^{*}=\min \left\{\varepsilon_{1}^{*}, \varepsilon_{2}^{*}\right\}$, we complete our proof.

In view of Section 2.1, Condition 1 of Theorem 4.2 is necessary to guarantee solvability of the regulator problem for the system in the absence of the saturation element. Under assumptions A1 and A2, Condition 2(a) is necessary for the existence of a linear stabilizing feedback for the system (3.1). The crucial condition for the solvability of the semi-global linear state feedback regulator problem is Condition 2(b), which is a sufficient condition. In section 5 we will discuss the necessity of Condition 2(b).

It is interesting to observe that if $\Gamma w=0$, then Condition $2(\mathrm{~b})$ of Theorem 4.2 is automatically satisfied. The following lemma examines the cases for which $\Gamma w=0$ holds.

Lemma 4.3: Consider Condition 3 of Theorem 4.2. $\Gamma w=0$ for all $w(0) \in \mathcal{W}_{0}$ if and only if $\mathcal{W}_{0} \subset<\operatorname{ker} \Gamma \mid S>$, where $<\operatorname{ker} \Gamma \mid S>$ is the unobservable subspace of the pair $(S, \Gamma)$.

Note that according to the sufficient conditions in the above theorem regulation is possible for arbitrary compact sets $\mathcal{W}_{0}$ if $\Gamma=0$. The following lemma specifies when this can happen.

Lemma 4.4: Consider Condition 2(b) of Theorem 4.2. $\Gamma=0$ if there exists a matrix $\Pi$ which solves the following matrix equations

$$
\left\{\begin{array}{l}
\Pi S=A \Pi+P \\
C \Pi+Q=0
\end{array}\right.
$$

\subsection{Dynamic Error Feedback}

The semi-global linear observer based error feedback regulator problem can be formulated as follows.

Definition 4.5: (Semi-Global Linear Observer Based Error Feedback Regulator Problem) Consider the system (3.1) and a compact set $\mathcal{W}_{0} \subset \mathbf{R}^{s}$. The semi-global error feedback regulator problem is defined as follows.

For any a priori given (arbitrarily large) bounded sets $\mathcal{X}_{0} \subset \mathbf{R}^{n}$ and $\mathcal{Z}_{0} \subset \mathbf{R}^{n+s}$, find, if possible, an error feedback law of the form

$$
\left\{\begin{array}{l}
{\left[\begin{array}{c}
\dot{\hat{x}} \\
\dot{\hat{w}}
\end{array}\right]=\left[\begin{array}{cc}
A & P \\
0 & S
\end{array}\right]\left[\begin{array}{l}
\hat{x} \\
\hat{w}
\end{array}\right]+\left[\begin{array}{c}
B \\
0
\end{array}\right] \sigma(u)+\left[\begin{array}{c}
L_{A} \\
L_{S}
\end{array}\right]\left(e-\left[\begin{array}{ll}
C & Q
\end{array}\right]\left[\begin{array}{l}
\hat{x} \\
\hat{w}
\end{array}\right]\right)} \\
u=F \hat{x}+G \hat{w}
\end{array}\right.
$$

such that 
1. The equilibrium $(x, \hat{x}, \hat{w})=(0,0,0)$ of

$$
\left\{\begin{array}{l}
\dot{x}=A x+B \sigma(F \hat{x}+G \hat{w})) \\
{\left[\begin{array}{c}
\dot{\hat{x}} \\
\dot{\hat{w}}
\end{array}\right]=\left[\begin{array}{cc}
A & P \\
0 & S
\end{array}\right]\left[\begin{array}{c}
\hat{x} \\
\hat{w}
\end{array}\right]+\left[\begin{array}{c}
B \\
0
\end{array}\right] \sigma(u)+\left[\begin{array}{l}
L_{A} \\
L_{S}
\end{array}\right]\left(\left[\begin{array}{ll}
C & Q
\end{array}\right]\left[\begin{array}{c}
x-\hat{x} \\
-\hat{w}
\end{array}\right]\right)}
\end{array}\right.
$$

is locally exponential stable with $\mathcal{X}_{0} \times \mathcal{Z}_{0}$ contained in its basin of attraction.

2. For all $(x(0), \hat{x}(0), \hat{w}(0)) \in \mathcal{X}_{0} \times \mathcal{Z}_{0}$ and $w(0) \in \mathcal{W}_{0}$, the solution of the closed-loop system satisfies

$$
\lim _{t \rightarrow \infty} e(t)=0 .
$$

Remark : We would like to emphasize that our definition of the semi-global error feedback regulator problem does not view the set of initial conditions of the plant and the initial conditions of the controller dynamics as given data. The set of given data consists of the models of the plant and the exosystem and the set of initial conditions for the exosystem. Therefore, the solvability conditions must be independent of the set of initial conditions of the plant, $\mathcal{X}_{0}$, and the set of initial conditions for the controller dynamics, $\mathcal{Z}_{0}$.

The solvability conditions for semi-global error feedback regulator problem are given in the following theorem.

Theorem 4.6; Consider the system (3.1) and the given compact set $\mathcal{W}_{0} \subset \mathbf{R}^{s}$. The semi-global error feedback regulator problem is solvable if

1. $(A, B)$ is stabilizable and $A$ has all eigenvalues in the closed left half plane. Moreover, the pair

$$
\left(\left[\begin{array}{ll}
C & Q
\end{array}\right],\left[\begin{array}{cc}
A & P \\
0 & S
\end{array}\right]\right)
$$

is detectable;

2. There exist matrices $\Pi$ and $\Gamma$ such that:

(a) They solve the following linear matrix equations :

$$
\left\{\begin{array}{l}
\Pi S=A \Pi+B \Gamma+P \\
C \Pi+Q=0
\end{array}\right.
$$

(b) There exists a $\delta>0$ and a $T \geq 0$ such that $\|\Gamma w\|_{\infty, \mathrm{T}} \leq 1-\delta$ for all $w$ with $w(0) \in \mathcal{W}_{0}$.

Proof: We prove this theorem by first explicitly constructing a family of linear observer based error feedback laws of the form (4.12), parameterized in $\varepsilon$, and then showing that for each pair of sets $\mathcal{X}_{0} \subset \mathbf{R}^{n}$ and $\mathcal{Z}_{0} \subset \mathbf{R}^{n+s}$, there exists an $\varepsilon^{*}>0$ such that for all $\varepsilon \in\left(0, \varepsilon^{*}\right.$, both items 1 and 2 in Definition 4.5 are indeed satisfied. The family of linear observer based error feedback laws we construct take the following form

$$
\left\{\begin{array}{l}
\dot{\hat{x}}=A \hat{x}+B \sigma(u)+P \hat{w}+L_{A} C(x-\hat{x})+L_{A} Q(w-\hat{w}) \\
\dot{\hat{w}}=S \hat{w}+L_{S} C(x-\hat{x})+L_{S} Q(w-\hat{w}) \\
u=-F(\varepsilon) \hat{x}+(F(\varepsilon) \Pi+\Gamma) \hat{w}
\end{array}\right.
$$


where $F(\varepsilon)$ is a state feedback gain matrix, parameterized in $\varepsilon$, chosen in such a way that $A-B F(\varepsilon)$ is Hurwitz and

$$
\begin{aligned}
& \|F(\varepsilon)\| \leq \alpha(\varepsilon) \\
& \left\|F(\varepsilon) e^{(A-B F(\varepsilon)) t}\right\|_{\infty} \leq \beta(\varepsilon) e^{-\gamma(\varepsilon) t}
\end{aligned}
$$

where $\alpha(\varepsilon), \beta(\varepsilon), \gamma(\varepsilon) \rightarrow 0$ as $\varepsilon \rightarrow 0$. The existence and the explicit construction of such an $F(\varepsilon)$ is established in Section 2.2. The matrices $L_{A}$ and $L_{S}$ are chosen such that the following matrix is Hurwitz,

$$
\bar{A}:=\left[\begin{array}{cc}
A-L_{A} C & P-L_{A} Q \\
-L_{S} C & S-L_{S} Q
\end{array}\right]
$$

The existence of such $L_{A}$ and $L_{S}$ is guaranteed by Condition 1 .

With this family of feedback laws, the closed-loop system consisting of the system (3.1) and the dynamic error feedback laws (4.16) can be written as,

$$
\left\{\begin{array}{l}
\dot{x}=A x+B \sigma(\Gamma \hat{w}-F(\varepsilon)(\hat{x}-\Pi \hat{w}))+P w \\
\dot{\hat{x}}=A \hat{x}+B \sigma(\Gamma \hat{w}-F(\varepsilon)(\hat{x}-\Pi \hat{w}))+P \hat{w}+L_{A} C(x-\hat{x})+L_{A} Q(w-\hat{w}) \\
\dot{\hat{w}}=S \hat{w}+L_{S} C(x-\hat{x})+L_{S} Q(w-\hat{w})
\end{array}\right.
$$

We then adopt the invertible change of state variable,

$$
\left\{\begin{array}{l}
\xi=x-\Pi w \\
\tilde{x}=x-\hat{x} \\
\tilde{w}=w-\hat{w}
\end{array}\right.
$$

and rewrite the closed loop system (4.20) as

$$
\left\{\begin{array}{l}
\dot{\xi}=A \xi+B \sigma(-F(\varepsilon) \xi+\Gamma w-\Gamma \tilde{w}+F(\varepsilon) \tilde{x}-F(\varepsilon) \Pi \tilde{w})+(A \Pi-\Pi S+P) w \\
\dot{\tilde{x}}=\left(A-L_{A} C\right) \tilde{x}+\left(P-L_{A} Q\right) \tilde{w} \\
\dot{\tilde{w}}=-L_{S} C \tilde{x}+\left(S-L_{S} Q\right) \tilde{w}
\end{array}\right.
$$

To show that item 1 of Definition 4.5 holds, we note that (4.12) is equal to (4.20) for $w=0$. Hence (4.22) reduces to

$$
\left\{\begin{array}{l}
\dot{\xi}=A \xi+B \sigma(-F(\varepsilon) \xi-\Gamma \tilde{w}+F(\varepsilon) \tilde{x}-F(\varepsilon) \Pi \tilde{w}) \\
\dot{\tilde{x}}=\left(A-L_{A} C\right) \tilde{x}+\left(P-L_{A} Q\right) \tilde{w} \\
\dot{\tilde{w}}=-L_{S} C \tilde{x}+\left(S-L_{S} Q\right) \tilde{w}
\end{array}\right.
$$

Recalling that the matrix $\bar{A}$, defined in (4.19), is Hurwitz and using (4.17), it readily follows from the last two equations of (4.23) that there exists a $T_{1} \geq 0$ such that, for all possible initial conditions $(\tilde{x}(0), \tilde{w}(0))$,

$$
\|\Gamma \tilde{w}\|_{\infty, \mathrm{T}_{1}} \leq \frac{1}{4},\|F(\varepsilon) \tilde{x}\|_{\infty, \mathrm{T}_{1}} \leq \frac{1}{4},\|F(\varepsilon) \Pi \tilde{w}\|_{\infty, \mathrm{T}_{1}} \leq \frac{1}{4} ; \forall \varepsilon \in(0,1]
$$


We next consider the first equation of (4.23). $\xi\left(T_{1}\right)$ belongs to a bounded set independent of $\varepsilon$ since $\xi(0)$ is bounded and since $\xi$ is determined via a linear differential equation with bounded input $\sigma(u)$. Hence there exists an $M_{1}$ such that for all possible initial conditions,

$$
\left\|\xi\left(T_{1}\right)\right\| \leq M_{1}, \forall \varepsilon \in(0,1]
$$

Let us now assume that, from time $T_{1}$ onwards, the saturation elements are nonexistent. In this case, the first equation of (4.23) can be written as

$$
\dot{\xi}=(A-B F(\varepsilon)) \xi+F(\varepsilon) \tilde{x}-B \Gamma \tilde{w}-B F(\varepsilon) \Pi \tilde{w}
$$

Since $\tilde{x} \rightarrow 0$ and $\tilde{w} \rightarrow 0$ exponentially with a decay rate independent of $\varepsilon$ as $t \rightarrow \infty$, it follows trivially from (4.17) and (4.18) that there exist an $\varepsilon_{1}^{*}>0$ and an $M_{2}>0$ such that, for all possible initial conditions and all $\varepsilon \in\left(0, \varepsilon_{1}^{*}\right]$,

$$
\int_{T_{1}}^{\infty}\left\|e^{\gamma(\varepsilon) \tau}[F(\varepsilon) \tilde{x}(\tau)-B \Gamma \tilde{w}(\tau)-B F(\varepsilon) \Pi \tilde{w}(\tau)]\right\| d \tau \leq M_{2}
$$

which in turn shows that, for $t \geq T_{1}$,

$$
\begin{aligned}
\|F(\varepsilon) \xi(t)\|= & \| F(\varepsilon) e^{(A-B F(\varepsilon)) t} \xi\left(T_{1}\right)+ \\
& \int_{T_{1}}^{t} F(\varepsilon) e^{(A-B F(\varepsilon))(t-\tau)}[F(\varepsilon) \tilde{x}(\tau)-\Gamma \tilde{w}(\tau)-F(\varepsilon) \Pi \tilde{w}(\tau)] \| \\
\leq & \beta(\varepsilon) M_{1}+\beta(\varepsilon) \int_{T_{1}}^{\infty}\left\|e^{\gamma(\varepsilon) \tau}[F(\varepsilon) \tilde{x}(\tau)-B \Gamma \tilde{w}(\tau)-B F(\varepsilon) \Pi \tilde{w}(\tau)]\right\| d \tau \\
\leq & \beta(\varepsilon)\left(M_{1}+M_{2}\right)
\end{aligned}
$$

Choosing $\varepsilon_{2}^{*} \in\left(0, \varepsilon_{1}^{*}\right]$ such that for all $\varepsilon \in\left(0, \varepsilon_{2}^{*}\right]$,

$$
\|F(\varepsilon) \xi\|_{\infty, \mathrm{T}_{1}} \leq \frac{1}{4}
$$

which, together with (4.24), shows that the system (4.23) will operate linearly after time $T_{1}$ and locally exponential stability of this linear system follows from the separation principle.

In summary, we have shown that there exists an $\varepsilon_{2}^{*}>0$ such that, for all $\varepsilon \in\left(0, \varepsilon_{2}^{*}\right]$, the equilibrium $(0,0,0)$ of the system $(4.23)$ is locally exponentially stable with $\left(\mathcal{X}_{0}, \mathcal{Z}_{0}\right)$ contained in its basin of attraction.

We now proceed to show that item 2 of Definition 4.5 also holds. To this end, we consider the closed-loop system (4.22). Recalling that the matrix $\bar{A}$ is Hurwitz, and using (4.17), it readily follows from the last two equations of (4.22) that there exists an $T_{2} \geq T$ such that, for all possible initial conditions $(\tilde{x}(0), \tilde{w}(0))$,

$$
\|\Gamma \tilde{w}\|_{\infty, \mathrm{T}_{2}} \leq \frac{\delta}{4},\|F(\varepsilon) \tilde{x}\|_{\infty, \mathrm{T}_{2}} \leq \frac{\delta}{4},\|F(\varepsilon) \Pi \tilde{w}\|_{\infty, \mathrm{T}_{2}} \leq \frac{\delta}{4} ; \forall \varepsilon \in(0,1]
$$

We next consider the first equation of (4.22). $\xi\left(T_{2}\right)$ belongs to a bounded set independent of $\varepsilon$ since $\xi(0)$ is bounded and since $\xi$ is determined via a linear differential equation with bounded inputs $\sigma(u)$ and $w$. Hence there exists an $M_{3}$ such that for all possible initial conditions,

$$
\left\|\xi\left(T_{2}\right)\right\| \leq M_{3}, \quad \forall \varepsilon \in(0,1]
$$

Let us now assume that, from time $T_{2}$ onwards, the equation (4.22) operates without the saturation elements. In view of Condition $2(\mathrm{a})$, with the saturation elements absent, the first equation of (4.22) in the absence of the saturation elements is equal to the first equation of (4.23) 
and hence also reduces to (4.26) after time $T_{2}$. Hence, using a similar argument as above, we can show that there exists an $\varepsilon_{3}^{*}>0$ such that, for all $\varepsilon \in\left(0, \varepsilon_{3}^{*}\right]$,

$$
\|F(\varepsilon) \xi\|_{\infty, \mathrm{T}_{2}}<\frac{\delta}{4}
$$

which, together with (4.29) and Condition 2(b), shows that the system (4.22) will operate linearly after time $T_{2}$ and the exponential stability of this linear system follows from the separation principle. Hence, $e(t)=C \xi(t) \rightarrow 0$ as $t \rightarrow 0$.

Finally, taking $\varepsilon^{*}=\min \left\{\varepsilon_{2}^{*}, \varepsilon_{3}^{*}\right\}$, we complete our proof.

As in the full-information feedback case, condition 1 is necessary to guarantee solvability of the error feedback regulator problem for the system in the absence of the saturation element. Condition $2(a)$ is necessary for the existence of linear feedbacks which semi-globally stabilize the system (3.1) which is subject to saturation. Clearly this time we also needed a detectability assumption. Finally, the crucial condition for the solvability of semi-global linear observer based error feedback regulator problem is Condition 2(b). This condition is sufficient.

Note the surprising fact that, except for the detectability assumption, these conditions are the same for the cases of full-information feedback and dynamic feedback on the basis of the error signal only.

\section{Necessary Conditions - Linear versus Nonlinear Regulator}

The semi-global state feedback regulator problem and semi-global error feedback regulator problems, as defined in Section 3, requires regulators with a linear structure. Namely linear state feedback laws or the implementation of linear state feedback laws utilizing linear observers are required for the output regulation. The sufficient conditions for the existence of such "linear" regulators were given in Section 3. In this section we examine the necessity of these conditions. The necessity issue must be examined in two fronts.

The first issue is to examine the necessity of the solvability conditions given in Section 3 for the existence of "linear" regulators. The second issue is to examine whether we can weaken the solvability conditions if we allow non-linear regulators in our definitions of the state and the error feedback regulator problem. It turns out that under certain mild conditions our solvability conditions for the existence of "linear" regulators are basically necessary. Moreover, these conditions cannot be weakened by allowing nonlinear regulators. We also make an interesting observation that whenever these mild conditions are violated, there might be nonlinear state feedbacks that achieve output regulation while no linear state feedbacks would do so.

The necessary condition for the existence of the semi-global state feedback regulator using a general nonlinear feedback law is given in the following theorem.

Theorem 5.1: Consider the plant and the exosystem (3.1). Let assumptions A1 and A2 hold. Assume that in the absence of input saturation, the linear state feedback regulator problem is solvable, i.e., there exist matrices $\Pi$ and $\Gamma$ which solve the linear matrix equations (2.6). Also assume that $(A, B, C, 0)$ is left-invertible and has no invariant zeros on the imaginary axis. Then, a necessary condition for the existence of a general, possibly nonlinear, state feedback laws that achieves semi-global regulation for (3.1) is that, for all $\varepsilon>0$, there exists a $T \geq 0$ such that

$$
\|\Gamma w(t)\|_{\infty, \mathrm{T}} \leq 1+\varepsilon, \forall w(0) \in \mathcal{W}_{0}
$$


Proof : The proof of the above theorem depends on some results described in the appendix. We will assume in the proof that the reader is familiar with these results. Consider the system (3.1) without the saturation element:

$$
\left\{\begin{array}{l}
\dot{x}=A x+B v+P w \\
\dot{w}=S w \\
e=C x+Q w
\end{array}\right.
$$

Suppose that we have some arbitrary nonlinear feedback $u=\alpha(x, w)$ which achieves regulation for the system (3.1). Then the feedback $v=\sigma(\alpha(x, w))$ will achieve regulation for the system (5.2).

We note that

$$
v=-F x+(\Gamma+F \Pi) w
$$

is a linear feedback which achieves regulation for the system (5.2). Moreover,

$$
v(t)-\Gamma w(t) \rightarrow 0 \text { as } t \rightarrow \infty
$$

We have two feedbacks which achieve regulation for the linear system (5.2). One is nonlinear and satisfies a certain amplitude constraint. The other feedback is a linear feedback of which we have no a priori knowledge regarding its amplitude. Our aim is to show that the linear feedback must necessarily satisfy an amplitude constraint asymptotically as $t \rightarrow \infty$. We define the difference between the two control inputs:

$$
s(t)=[-F x+(\Gamma+F \Pi) w](t)-[\sigma(\alpha(x, w))](t)
$$

Suppose Condition 2(b) is not true. In that case there exists $\left\{t_{n}\right\}_{n=1}^{\infty}$ and $\delta>0$ such that $t_{n} \rightarrow \infty$ as $n \rightarrow \infty$ and $\left\|\Gamma w\left(t_{n}\right)\right\|>1+2 \delta$ for all $n$. Given the differential equation for $w$ it is not difficult to see that this implies that there exists an $\varepsilon>0$ such that for all $n$ and all $t \in\left[t_{n}, t_{n}+\varepsilon\right]$ we have $\left\|\Gamma w\left(t_{n}\right)\right\|>1+\delta$.

If we look at our definition for $s$, we see that the first component asymptotically converges to $\Gamma w$. The second term is bounded by 1 . Combining this with the property for $\Gamma w$ we just derived it is easily seen that the vector-valued signal $s$ has a component $s_{i}$ for which we have, for all $n$, either

$$
\text { for all } t \in\left[t_{n}, t_{n}+\varepsilon\right]: \quad s_{i}(t)>\delta
$$

or

$$
\text { for all } t \in\left[t_{n}, t_{n}+\varepsilon\right]: \quad s_{i}(t)<-\delta
$$

In other words $s \in \mathcal{P}$ as defined in definition A.3. On the other hand if we apply $s$ to the system:

$$
\left\{\begin{array}{l}
\dot{x}=A x+B s \\
e=C x
\end{array}\right.
$$

with zero initial conditions then we have $e(t) \rightarrow 0$ (since both the linear feedback and the nonlinear saturating feedback achieve regulation).

Combined, the last two results yield a contradiction by applying theorem A.4. This implies that Condition 2(b) of theorem 4.2 is satisfied. Thus we find that the existence of a, possibly nonlinear, feedback achieving regulation for the system (3.1) implies that the conditions of theorem 4.2 are satisfied. 
Remark : The necessary conditions given in Theorem 5.1 are slightly different from the sufficient solvability conditions given in Theorem 4.2. Namely, Condition (5.1) of Theorem 5.1 and Condition $2(b)$ of Theorem 4.2 are not exactly the same, although they are almost equal. Hence, one can conclude that under Assumptions $A 1$ and that $(A, B, C, 0)$ is left invertible and has no invariant zeros on the imaginary axis, if the semi-global state feedback regulator problem is solvable by a nonlinear feedback regulator it is generally solvable by the linear feedback regulator.

An interesting question is whether one can weaken the necessary condition given in Theorem 5.1, if $(A, B, C, 0)$ is not left invertible and/or has invariant zeros in the $j w$ axis. The following example shows that, in fact, this is the case. More significantly, this example shows that if $(A, B, C, 0)$ is not left invertible, nonlinear feedbacks might achieve semi-global regulation while no linear feedback can do so.

Example 5.2: Consider the following system:

$$
\left\{\begin{array}{l}
\dot{x}=\left(\begin{array}{ccc}
-1 & 1 & 2 \\
0 & -2 & 0 \\
0 & 0 & -1
\end{array}\right) x+\left(\begin{array}{ccc}
1 & 0 & -1 \\
0 & 1 & 1 \\
-1 & 1 & 0
\end{array}\right) \sigma(u) \\
\dot{w}=\left(\begin{array}{ll}
0 & 0 \\
0 & 0
\end{array}\right) w \\
e=\left(\begin{array}{lll}
0 & 1 & 0 \\
0 & 0 & 1
\end{array}\right) x+\quad\left(\begin{array}{cc}
-0.5 & 0 \\
0 & -1
\end{array}\right) w
\end{array}\right.
$$

with $w(0) \in \mathcal{W}_{0}$ where

$$
\mathcal{W}_{0}=\text { convex hull }\left\{\left(\begin{array}{l}
2 \\
0
\end{array}\right),\left(\begin{array}{l}
0 \\
2
\end{array}\right),\left(\begin{array}{l}
2 \\
2
\end{array}\right)\right\}
$$

It is straightforward to show that in the absence of input saturation the linear state feedback regulator is solvable. In fact, the matrics $\Pi$ and $\Gamma$ that solve the linear matrix equations (2.6) are given by,

$$
\Pi=\left(\begin{array}{cc}
\pi_{11} & \pi_{12} \\
0.5 & 0 \\
0 & 1
\end{array}\right), \Gamma=\left(\begin{array}{cc}
0.5 \pi_{11}+0.25 & 0.5 \pi_{12}-1.5 \\
0.5 \pi_{11}+0.25 & 0.5 \pi_{12}-0.5 \\
-0.5 \pi_{11}+0.75 & -0.5 \pi_{12}+0.5
\end{array}\right)
$$

where $\pi_{11}$ and $\pi_{12}$ are any real numbers.

In the presence of input saturation, however, the sufficient conditions of Theorem 4.2 are not satisfied. More specifically, Condition 2(b) of Theorem 4.2 cannot be satisfied. Hence, the design procedure developed in Section 4 cannot be applied to this example. It is also evident that the necessary condition (5.1) cannot be satisfied either. However, since $(A, B, C, 0)$ in the given plant and the exosystem (5.5) is not left invertible, this does not implies that the there does not exist state feedback laws that achieve semi-global output regulation for (5.5). In fact, in what follows, we will establish the following two facts for the plant and the exosystem (5.5),

1. There exist nonlinear feedbacks that achieve semi-global output regulation. This would immediately imply that the necessary condition (5.1) given in Theorem 5.1 can be weakened if $(A, B, C, 0)$ is not left invertible; 
2. There exist no linear state feedbacks that can achieve semi-global output regulation. This establishes an important result. That is, if $(A, B, C, o)$ is not left invertible, the semi-global output regulation might be achieved via nonlinear feedbacks while no linear feedbacks can do so.

As the plant is already asymptotically stable, let us consider a nonlinear feedback of only the exosystem state of the form,

$$
u=\left(\begin{array}{c}
0.5 \\
0.5 \\
-0.5
\end{array}\right) f(w)+\left(\begin{array}{cc}
0.5 & -0.5 \\
0.5 & 0.5 \\
0.5 & -0.5
\end{array}\right) w
$$

and

$$
f(w)=(1-\alpha-\beta)\left(\begin{array}{ll}
-1 & 0
\end{array}\right) w
$$

where $\alpha, \beta \geq 0$ are such that $\alpha+\beta \leq 1$ and:

$$
w=\alpha\left(\begin{array}{l}
2 \\
0
\end{array}\right)+\beta\left(\begin{array}{l}
0 \\
2
\end{array}\right)+(1-\alpha-\beta)\left(\begin{array}{l}
2 \\
2
\end{array}\right)
$$

Note that for any $w(0) \in \mathcal{W}_{0}, w \equiv w(0)$.

Clearly since $\alpha$ and $\beta$ depend on $w$, the function $f$ is nonlinear and hence this compensator is nonlinear.

We study the following system:

$$
\left\{\begin{array}{l}
\dot{x}=\left(\begin{array}{ccc}
-1 & 1 & 2 \\
0 & -2 & 0 \\
0 & 0 & -1
\end{array}\right) x+\left(\begin{array}{l}
1 \\
0 \\
0
\end{array}\right) v_{1}+\left(\begin{array}{ll}
0 & 0 \\
1 & 0 \\
0 & 1
\end{array}\right) v_{2} \\
\dot{w}=\left(\begin{array}{cc}
0 & 0 \\
0 & 0
\end{array}\right) w \\
e=\left(\begin{array}{lll}
0 & 1 & 0 \\
0 & 0 & 1
\end{array}\right) x+\left(\begin{array}{cc}
-0.5 & 0 \\
0 & -1
\end{array}\right) w
\end{array}\right.
$$

Note that if we choose:

$$
\sigma(u)=\left(\begin{array}{c}
0.5 \\
0.5 \\
-0.5
\end{array}\right) v_{1}+\left(\begin{array}{cc}
0.5 & -0.5 \\
0.5 & 0.5 \\
0.5 & -0.5
\end{array}\right) v_{2}
$$

then we get the same closed loop system for (5.7) as in (5.5). Hence, we can try to solve the regulator problem for the system (5.7) subject to that $v_{2}$ and $v_{1}$ must be chosen such that (5.8) is solvable. Note that if we study the regulator problem for (5.7) then $v_{1}$ does not affect $e$ and hence can be ignored. Hence we obtain the regulator problem for the system with input $v_{2}$. The transfer matrix from $v_{2}$ to $e$ is left-invertible. Therefore we know that $v_{2}$ when achieving regulation has a unique asymptotic behavior (except for some freedom as described in theorem A.4 which cannot change the maximal amplitude). We find by solving the regulator equations that $v_{2}$ is equal to $w$ at least asymptotically. However for all $w(0) \in \mathcal{W}_{0}$, equation (5.8) must be solvable. For

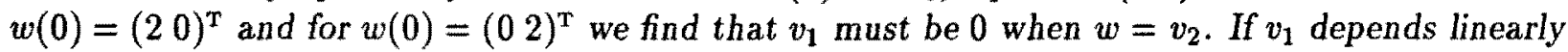
on $w$ then for $w(0)=\left(\begin{array}{ll}2 & 2\end{array}\right)^{\mathrm{T}}$ we must also choose $v_{1}=0$ when $w=v_{2}$. However in that case $(5.8)$ is not solvable and hence there exists no linear function from $w$ to $v_{1}$ such that regulation can be achieved for all $w(0) \in \mathcal{W}_{0}$. On the other hand, $v_{1}=f(w)$ and $v_{2}=w$ results in a solvable 


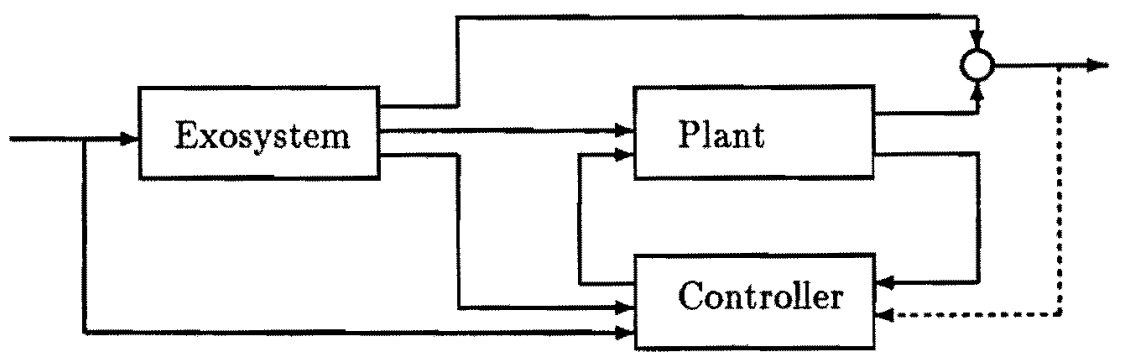

Figure 6.1: Configuration of a generalized regulator

equation (5.8) for all possible choices of $w(0) \in \mathcal{W}_{0}$. Moreover, the controller (5.6) is the unique solution. Therefore it is straightforward to check that the nonlinear controller (5.6) indeed solves the regulator problem for (5.5).

Remark : We can pose similar questions regarding the semi-global error feedback regulator problem. Again, there is the question whether the conditions of theorem 4.6 are actually necessary for the solvability of the semi-global error feedback regulator problem. But theorem 5.1 basically resolves this question since the conditions which are necessary for the full-information feedback are clearly also necessary for the case of output feedback on the basis of the error signal only. The only additional assumption we made in theorem 4.6 is the detectability assumption which is clearly necessary for the stabilization of our system.

\section{Generalized Semi-Global Linear Feedback Regulator Problems}

We have formulated the semi-global linear feedback regulator problems for linear systems subject to input saturation following the traditional formulation of linear regulator problems where the exosystem is autonomous. As a result, the disturbances and the references generated by the exosystem contain only the frequency components of the exosystem. In an effort to broaden the class of disturbance and reference signals, we formulate in this section the generalized semi-global linear feedback regulator problem, for which an external driving signal to the exosystem is included. More specifically, we consider a multivariable system with inputs that are subject to saturation together with an exosystem that generates disturbance and reference signals as described by the following system

$$
\left\{\begin{array}{l}
\dot{x}=A x+B \sigma(u)+P w \\
\dot{w}=S w+r \\
\epsilon=C x+Q w
\end{array}\right.
$$

where $x \in \mathbf{R}^{n}, w \in \mathbf{R}^{s}, u \in \mathbf{R}^{m}, e \in \mathbf{R}^{p}, r \in \mathcal{C}^{0}$ is an external signal to the exosystem, and $\sigma$ is a vector-valued saturation function as defined by (3.2).

The generalized semi-global linear state feedback regulator problem and the generalized error feedback regulator problem are formulated as follows. 
Definition 6.1: (Generalized Semi-Global Linear State Feedback Regulator Problem) Consider the system (6.1), a compact set $\mathcal{W}_{0} \subset \mathbf{R}^{s}$ and a compact set $\mathcal{R} \subset \mathcal{C}^{0}$. The problem of generalized semi-global linear state feedback regulator problem is defined as follows.

For any a priori given (arbitrarily large) bounded set $\mathcal{X}_{0} \subset \mathbf{R}^{n}$, find, if possible, a linear static feedback law $u=F x+G w+H r$, such that

1. The equilibrium $x=0$ of

$$
\dot{x}=A x+B \sigma(F x)
$$

is locally exponentially stable with $\mathcal{X}_{0}$ contained in its basin of attraction;

2. For all $x(0) \in \mathcal{X}_{0}, w(0) \in \mathcal{W}_{0}$ and $r \in \mathcal{R}$, the solution of the closed-loop system satisfies

$$
\lim _{t \rightarrow \infty} e(t)=0
$$

Definition 6.2: (Generalized Semi-Global Linear Observer Based Error Feedback Regulator Problem) Consider the system (6.1) and two compact sets $\mathcal{W}_{0} \subset \mathbf{R}^{s}$ and $\mathcal{R} \subset \mathcal{C}^{0}$. The semi-global error feedback regulator problem is defined as follows.

For any a priori given (arbitrarily large) bounded sets $\mathcal{X}_{0} \subset \mathbf{R}^{n}$ and $\mathcal{Z}_{0} \subset \mathbf{R}^{n+s}$, find, if possible, a linear observer based error feedback law of the form:

$$
\left\{\begin{array}{l}
{\left[\begin{array}{c}
\dot{\hat{x}} \\
\dot{\hat{w}}
\end{array}\right]=\left[\begin{array}{cc}
A & P \\
0 & S
\end{array}\right]\left[\begin{array}{c}
\hat{x} \\
\hat{w}
\end{array}\right]+\left[\begin{array}{c}
B \\
0
\end{array}\right] \sigma_{h}(u)+\left[\begin{array}{c}
L_{A} \\
L_{S}
\end{array}\right]\left(e-\left[\begin{array}{ll}
C & Q
\end{array}\right]\left[\begin{array}{l}
\hat{x} \\
\hat{w}
\end{array}\right]\right)} \\
u=F \hat{x}+G \hat{w}+H r
\end{array}\right.
$$

such that

1. The equilibrium $(x, \hat{x}, \hat{w})=(0,0,0)$ of

$$
\left\{\begin{array}{l}
\left.\dot{x}=A x+B \sigma_{h}(F \hat{x}+G \hat{w})\right) \\
{\left[\begin{array}{c}
\dot{\hat{x}} \\
\hat{\hat{w}}
\end{array}\right]=\left[\begin{array}{cc}
A & P \\
0 & S
\end{array}\right]\left[\begin{array}{l}
\hat{x} \\
\hat{w}
\end{array}\right]+\left[\begin{array}{c}
B \\
0
\end{array}\right] \sigma_{h}(u)+\left[\begin{array}{c}
L_{A} \\
L_{S}
\end{array}\right]\left(\left[\begin{array}{ll}
C & Q
\end{array}\right]\left[\begin{array}{c}
x-\hat{x} \\
-\hat{w}
\end{array}\right]\right)}
\end{array}\right.
$$

is locally exponentially stable with $\mathcal{X}_{0} \times \mathcal{Z}_{0}$ contained in its basin of attraction;

2. For all $(x(0), \hat{x}(0), \hat{w}(0)) \in \mathcal{X}_{0} \times \mathcal{Z}_{0}, w(0) \in \mathcal{W}_{0}$, and all $r \in \mathcal{R}$, the solution of the closed-loop system satisfies

$$
\lim _{t \rightarrow \infty} e(t)=0
$$

Remark : We would like to emphasize here that our definition of the generalized semi-global linear state feedback [respectively, linear observer based error feedback] regulator problem does not view the set of initial conditions of the plant as given data. The set of given data consists of the models of the plant and the exosystem, the set of initial conditions for the exosystem and the set of external input to the exosystem. Moreover, the generalized semi-global linear state feedback [respectively, error feedback] regulator problem reduces to the semi-global linear state feedback [respectively, error feedback] regulator problem as formulated in Definition 4.1 [respectively, Definition 4.5] when the external input $r$ to the exosystem is nonexistent. 
Remark : We would also like to emphasize that unlike the traditional regulator problem where all interesting cases arise when the poles of the exosystem are in the closed right half plane, for the generalized regulator problem, there are interesting cases even when the exosystem is asymptotically stable.

We will give solvability conditions for the above two problems. For clarity, we present these solvability conditions in two separate subsections, one for the state feedback case and the other for the error feedback case. As a special case of the generalized semi-global linear feedback regulator problems, the solvability conditions for semi-global linear feedback tracking problems for a chain of integrators are thus obtained readily. The same set of solvability conditions for the global state feedback tracking problem for a chain of integrators were given earlier in [18], where nonlinear feedbacks were resorted to.

\subsection{Static State Feedback}

The solvability conditions for generalized semi-global linear state feedback regulator problem is given in the following theorem.

Theorem 6.3: Consider the system (6.1) and given compact sets $\mathcal{W}_{0} \subset \mathbf{R}^{s}$ and $\mathcal{R} \subset \mathcal{C}^{0}$. The generalized semi-global linear state feedback regulator problem is solvable if

1. $(A, B)$ is stabilizable and $A$ has all its eigenvalues in the closed left half plane.

2. There exist matrices $\Pi$ and $\Gamma$ such that:

(a) They solve the following linear matrix equations:

$$
\left\{\begin{array}{l}
\Pi S=A \Pi+B \Gamma+P \\
C \Pi+Q=0
\end{array}\right.
$$

(b) For each $r \in \mathcal{R}$, there exists a function $\tilde{r} \in \mathcal{C}^{0}$ such that $\Pi r=B \tilde{r}$.

(c) There exists a $\delta>0$ and a $T \geq 0$ such that $\|\Gamma w+\tilde{r}\|_{\infty, \mathrm{T}} \leq 1-\delta$ for all $w$ with $w(0) \in \mathcal{W}_{0}$ and all $r \in \mathcal{R}$.

Remark : We would like to make the following observations on the solvability conditions as given in the above theorem:

1. As expected, the solvability conditions for the generalized semi-global linear state feedback regulator problem as given in the above theorem reduces to those for the semi-global linear state feedback regulator problem as formulated in Definition 4.1 when the external input to the exosystem is nonexistent.

2. If $\operatorname{Im} \Pi \subset \operatorname{Im} B$, then Condition $2(b)$ is automatically satisfied any given set $\mathcal{R}$.

3. If $\operatorname{Im} \Pi \cap \operatorname{Im} B=\{0\}$, then Condition $2(b)$ can never be satisfied for any given $\mathcal{R}$ except for $\mathcal{R}=\{0\}$.

Proof of Theorem 6.3: The proof of this theorem is similar, mutatis mutandis, to that of Theorem 4.2. As in the proof of Theorem 4.2, we prove this theorem by first constructing a family of linear static state feedback laws, parameterized in $\varepsilon$, and then showing that for each given set $\mathcal{X}_{0}$, there 
exists an $\varepsilon^{*}>0$ such that for all $\varepsilon \in\left(0, \varepsilon^{*}\right]$, both items 1 and 2 of Definition 6.1 hold. The family of linear static state feedback laws we construct takes the following form

$$
u=-F(\varepsilon) x+(F(\varepsilon) \Pi+\Gamma) w+\tilde{r}
$$

The rest of the proof is the same as that of Theorem 4.2 except that (4.8) takes the following slightly different form

$$
\dot{\xi}=A \xi+B(\sigma(u)-\Gamma w-\tilde{r})
$$

As a corollary to Theorem 6.3, we give the solvability conditions for the semi-global linear state feedback restricted tracking problem. It is interesting to note that the same solvability condition was given by Teel [18] for the global nonlinear state feedback restricted tracking problem for a chain of integrators.

Corollary 6.4: Consider a system consisting of a chain of integrators

$$
\left\{\begin{array}{l}
\dot{x}_{i}=x_{i+1}, \quad i=1,2, \cdots, n-1 \\
\dot{x}_{n}=\sigma(u) \\
y=x_{1}
\end{array}\right.
$$

Let a desired reference trajectory be given by $y_{d} \in \mathcal{C}^{n}$. Assume that there exists a $T \geq 0$ and a $\delta>0$ such that $\left|y_{d}^{(n)}\right| \leq 1-\delta$ for all $t \geq T$. Then the reference signal $y_{d}$ can be semi-giobally tracked by $y$ via linear static state feedback. More specifically, for any given (arbitrarily large) bounded set $\mathcal{X}_{0} \subset \mathbb{R}^{n}$, there exists a linear static state feedback law $u=-\sum_{i=1}^{n} F_{i} x_{i}+y_{d}^{(n)}$ such that

1. The equilibrium $\left(x_{1}, x_{2}, \cdots, x_{n}\right)=(0,0, \cdots, 0)$ of

$$
\left\{\begin{array}{l}
\dot{x}_{i}=x_{i+1}, i=1,2, \cdots, n-1 \\
\dot{x}_{n}=\sigma\left(-\sum_{i=1}^{n} F_{i} x_{i}\right)
\end{array}\right.
$$

is locally exponentially stable with $\mathcal{X}_{0}$ contained in its basin of attraction;

2. For any $x(0) \in \mathcal{X}_{0}$, the solution of the closed-loop system satisfies

$$
\lim _{t \rightarrow \infty} y(t)=y_{d}(t)
$$

Proof : The desired reference trajectory can be modeled as that of the following exogenous system

$$
\left\{\begin{array}{l}
\dot{w}_{i}=w_{i+1}, w_{i}(0)=y_{d}^{(i-1)}(0), i=1,2, \cdots, n-1 \\
\dot{w}_{n}=y_{d}^{(n)}, w_{n}(0)=y_{d}^{(n-1)}(0)
\end{array}\right.
$$


and hence the tracking problem can be cast into a generalized linear state feedback regulator problem as defined in definition 6.1 , with $P=0, \mathcal{R}=\left\{\left[00 \cdots 0 r_{n}(t)\right]^{\prime} \in \mathcal{C}^{0}:\left|r_{n}^{(n)}\right| \leq 1-\delta\right\}$, $\mathcal{W}_{0}:=\left\{\left(y_{d}(0), y_{d}^{\prime}(0), \cdots, y_{d}^{(n-1)}(0)\right)\right\}$ and

$$
A=S=\left[\begin{array}{ccccc}
0 & 1 & 0 & \cdots & 0 \\
0 & 0 & 1 & \cdots & 0 \\
\vdots & \vdots & \vdots & \ddots & \vdots \\
0 & 0 & 0 & \cdots & 1 \\
0 & 0 & 0 & \cdots & 0
\end{array}\right], C=Q=\left[\begin{array}{c}
1 \\
0 \\
\vdots \\
0 \\
0
\end{array}\right]^{\prime}
$$

Clearly, $I I=I$ and $\Gamma=0$ solve the matrix equations (6.7). Condition 1 of Theorem 6.3 is clearly satisfied, while Condition 2 is also satisfied trivially with $\tilde{r}=y_{d}^{(n)}$. The results of this corollary thus follow from that of Theorem 6.3.

Note that we could have modelled the exogenous system with zero dynamical order and $r=$ $w=y_{d}$, but then the matching Condition 2(b) of Theorem 6.3 would not be satisfied. Our model explicitly incorporates that $y_{d} \in \mathcal{C}^{n}$ and then results in a solvable regulator problem.

\subsection{Dynamic Error Feedback}

The solvability conditions for generalized semi-global linear observer based error feedback regulator problem is given in the following theorem.

Theorem 6.5: Consider the system (6.1) and the given compact sets $\mathcal{W}_{0} \subset \mathbf{R}^{s}$ and $\mathcal{R} \subset \mathcal{C}^{0}$. Let $l=n+s$. The semi-global linear observer based error feedback regulator problem is solvable if

1. $(A, B)$ is stabilizable and $A$ has all its eigenvalues in the closed left half plane. Moreover, the pair

$$
\left(\left[\begin{array}{ll}
C & Q
\end{array}\right],\left[\begin{array}{cc}
A & P \\
0 & S
\end{array}\right]\right)
$$

is detectable;

2. There exist matrices $\Pi$ and $\Gamma$ such that:

(a) They solve the following linear matrix equations :

$$
\left\{\begin{array}{l}
\Pi S=A \Pi+B \Gamma+P \\
C \Pi+Q=0
\end{array}\right.
$$

(b) For each $r \in \mathcal{R}$, there exists a function $\tilde{r}(t) \in \mathcal{C}^{0}$ such that $\Pi r(t)=B \tilde{r}(t)$ for all $t \geq 0$.

(c) There exist a $\delta>0$ and a $T \geq 0$ such that $\|\Gamma w+\tilde{r}\|_{\infty, \mathrm{r}} \leq 1-\delta$ for all $w$ with $w(0) \in \mathcal{W}_{0}$ and all $r \in \mathcal{R}$.

Remark : As expected, the solvability conditions for the generalized semi-global linear observer based error feedback regulator problem as given in the above theorem reduces to those for the semi-global linear observer based error feedback regulator problem as formulated in Definition 4.5 when the external input to the exosystem is nonexistent. 
Proof of Theorem 6.5: The proof of this theorem is similar, mutatis mutandis to that of Theorem 4.6. As in the proof of Theorem 4.6, we prove this theorem by first constructing a family of linear observer based error feedback laws, parameterized in $\varepsilon$, and then showing that both items 1 and 2 of Definition 6.2 indeed hold. The family of linear observer based error feedback laws we construct takes the following form

$$
\left\{\begin{array}{l}
\dot{\hat{x}}=A \hat{x}+B \sigma(u)+P \hat{w}+L_{A} C(x-\hat{x})+L_{A} Q(w-\hat{w}) \\
\dot{\hat{w}}=S \hat{w}+L_{S} C(x-\hat{x})+L_{S} Q(w-\hat{w}) \\
u=-F(\varepsilon) \hat{x}+(F(\varepsilon) \Pi+\Gamma) \hat{w}+\tilde{r}
\end{array}\right.
$$

where $L_{A}$ and $L_{S}$ are such that the following matrix is Hurwitz,

$$
\bar{A}:=\left[\begin{array}{cc}
A-L_{A} C & P-L_{A} Q \\
-L_{S} C & S-L_{S} Q
\end{array}\right]
$$

The rest of the proof is the same as that of Theorem 4.6 except that (4.22) takes the following form instead

$$
\left\{\begin{array}{l}
\dot{\xi}=A \xi+B \sigma(-F(\varepsilon) \xi+\Gamma w-\Gamma \tilde{w}+F(\varepsilon) \tilde{x}-F(\varepsilon) \Pi \tilde{w}+\tilde{r})+(A \Pi-\Pi S+P) w-\Pi r \\
\dot{\tilde{x}}=\left(A-L_{A} C\right) \tilde{x}+\left(P-L_{A} Q\right) \tilde{w} \\
\dot{\tilde{w}}=-L_{S} C \tilde{x}+\left(S-L_{S} Q\right) \tilde{w}
\end{array}\right.
$$

Remark : From the above proof of Theorem 6.5, we note that the linear state feedback law (6.8) interconnected with any exponentially stable observer (where $x$ and $w$ are replaced by their respective estimates which converge exponentially to the real $x$ and $w$ as $t \rightarrow \infty$ ) will solve the semi-global error feedback regulator problem.

As a corollary to Theorem 6.5 , we obtain the solvability conditions for the semi-global linear observer based output feedback restricted tracking problem.

Corollary 6.6: Consider the system of a chain of integrators

$$
\left\{\begin{array}{l}
\dot{x}_{i}=x_{i+1}, i=1,2, \cdots, n-1 \\
\dot{x}_{n}=\sigma(u) \\
y=x_{1}
\end{array}\right.
$$

Let a desired reference trajectory be given by $y_{d} \in \mathcal{C}^{n}$. If there exist a $T \geq 0$ and a $\delta>0$ such that $\left\|y_{d}^{(n)}\right\|_{\infty, \mathrm{r}} \leq 1-\delta$. The reference signal $y_{d}(t)$ can then be semi-globally tracked by $y$ via linear observer based output feedback.

More specifically, for any given (arbitrarily large) bounded sets $\mathcal{X}_{0} \subset \mathbf{R}^{n}$ and $\mathcal{Z}_{0} \subset \mathbf{R}^{n}$, there exists a linear observer based output feedback law

$$
\left\{\begin{array}{l}
\dot{\hat{x}}=A \hat{x}+B \sigma(u)+L(y-C \hat{x}) \\
u=-F \hat{x}+y_{d}^{(n)}
\end{array}\right.
$$

such that 
1. The equilibrium $\left(x_{1}, x_{2}, \cdots, x_{n}, \hat{x}\right)=(0,0, \cdots, 0,0)$ of

$$
\left\{\begin{array}{l}
\dot{x}_{i}=x_{i+1}, \quad i=1,2, \cdots, n-1 \\
\dot{x}_{n}=\sigma\left(-F \hat{x}+y_{d}^{(n)}\right) \\
\dot{\hat{x}}=A \hat{x}+B \sigma\left(-F \hat{x}+y_{d}^{(n)}\right)+L(y-C \hat{x})
\end{array}\right.
$$

is locally exponentially stable with $\mathcal{X}_{0} \times \mathcal{Z}_{0}$ contained in its basin of attraction;

2. For any $(x(0), \hat{x}(0)) \in \mathcal{X}_{0} \times \mathcal{Z}_{0}$, the solution of the closed-loop system satisfies

$$
\lim _{t \rightarrow \infty} y(t)=y_{d}(t)
$$

Proof: The desired reference trajectory can be modeled as that of the following exosystem

$$
\left\{\begin{array}{l}
\dot{w}_{i}=w_{i+1}, w_{i}(0)=y_{d}^{(i-1)}(0), i=1,2, \cdots, n-1 \\
\dot{w}_{n}=y_{d}^{(n)}, w_{n}(0)=y_{d}^{(n-1)}(0)
\end{array}\right.
$$

and hence the output feedback tracking problem can be cast into a generalized semi-global linear observer-based error feedback regulator problem as defined in definition 6.2 , with $P=0, \mathcal{R}=$ $\left\{\left[00 \cdots 0 r_{n}(t)\right]^{\prime} \in \mathcal{C}^{0}:\left|r_{n}^{(n)}\right| \leq 1-\delta\right\}, W_{0}=\left\{\left(y_{d}(0), y_{d}^{\prime}(0), \cdots, y_{d}^{(n-1)}(0)\right)\right\}$ and

$$
A=S=\left[\begin{array}{ccccc}
0 & 1 & 0 & \cdots & 0 \\
0 & 0 & 1 & \cdots & 0 \\
\vdots & \vdots & \vdots & \ddots & \vdots \\
0 & 0 & 0 & \cdots & 1 \\
0 & 0 & 0 & \cdots & 0
\end{array}\right], C=Q=\left[\begin{array}{c}
1 \\
0 \\
\vdots \\
0 \\
0
\end{array}\right]^{\prime}
$$

Clearly, $\Pi=I$ and $\Gamma=0$ solve the matrix equations (6.11) and hence Condition 2 of Theorem 6.5 is satisfied trivially with $\tilde{r}=y_{d}^{(n)}$. We note Condition 1 is not satisfied since the pair

$$
\left(\left[\begin{array}{ll}
C & Q
\end{array}\right],\left[\begin{array}{cc}
A & P \\
0 & S
\end{array}\right]\right)
$$

is not detectable. From the proof of Theorem 6.5, we note that the linear state feedback law (6.8) interconnected with any stable observer will solve the semi-global error feedback regulator problem. We know the disturbance $w=\left[\begin{array}{llll}y_{d} & \dot{y}_{d} & \cdots & y_{d}^{(n-1)}\end{array}\right]$ exactly and hence we only need an asymptotic estimate of the state $x$. To this end, we build a state estimator to estimate the state $x$ using the measurement $y=e+y_{d}$. This estimator takes the form

$$
\dot{\hat{x}}=A \hat{x}+B \sigma(u)+L(y-C \hat{x})
$$

where $L$ is such that the matrix $(A-L C)$ is Hurwitz. We then implement the state feedback law as given in Corollary 6.4 with the estimated state $\hat{x}$ and obtain the following output feedback law that solves the output feedback restricted tracking problem

$$
\left\{\begin{array}{l}
\dot{\hat{x}}=A \hat{x}+B \sigma(u)+L(y-C \hat{x}) \\
u=-F \hat{x}+y_{d}^{(n)}
\end{array}\right.
$$

This completes the proof of Corollary 6.6. 


\section{Conclusions}

In this paper we have studied the regulator problem for linear systems subject to input saturation. We have shown that the semi-global setting is natural for this problem.

Under a mild assumption we have given necessary and sufficient conditjons for the existence of a linear state feedback which achieves regulation. Moreover, we have shown that nonlinear compensators cannot do better in our semi-global setting.

We also studied the error feedback regulator problem. Under the same mild assumption we again obtain necessary and sufficient conditions for the existence of a error feedback which achieves regulation. The error feedbacks we used here have a linear observer-based structure. We also show that nonlinear, dynamic compensators cannot do better.

If this assumption is not satisfied then our conditions for both state and error feedback are still sufficient for the existence of a feedback with a linear structure which achieves regulation. However, nonlinear feedbacks might do better.

\section{Appendix A: Uniqueness of Asymptotic Behaviour of the Input}

In this section we will prove that under rather weak assumptions the asymptotic behavior of the input is unique given that the output of the system tracks a certain reference signal. This is a result which is used in the proof of theorem 5.1.

The following theorem from [2] is a very powerful tool in the analysis of the asymptotic behavior of the signals.

Theorem A.1: Let $R, S, V, W$ be matrix valued polynomials. Define $k$ as the maximal degree of the four polynomials. The following conditions are equivalent:

1. For any vector-valued function $u$ on $[0, \infty)$ which is $k$ times differentiable the following conditions:

$$
\begin{array}{ll}
r:=R\left(\frac{d}{d t}\right) u: & r(t)=0 \quad \forall t \\
s:=S\left(\frac{d}{d t}\right) u: & s(t) \rightarrow 0 \quad \text { as } t \rightarrow \infty \\
v:=V\left(\frac{d}{d t}\right) u: & \sup _{t} v(t)<\infty
\end{array}
$$

imply

$$
w:=W\left(\frac{d}{d t}\right) u: \quad w(t) \rightarrow 0 \text { as } t \rightarrow \infty
$$

2. Define the following equation:

$$
M(s) R(s)+N(s) S(s)+L(s) V(s)=W(s)
$$

Then we have:

(a) For all $\alpha \in \mathbb{C}^{+}$there exist rational matrices $M, N, L$ which do not have poles in $\alpha$ satisfying (A.1).

(b) For all $\alpha \in \mathbb{C}^{0}$ there exist rational matrices $M, N, L$ which do not have poles in $\alpha$ satisfying (A.1) and, moreover, $L(\alpha)=0$. 
(c) there exist rational matrices $M, N, L$ satisfying (A.1) and, moreover, $N$ is a proper and $L$ is a strictly proper rational matrix.

Remark : Note that the proof in [2] is set in a distributional setting. Hence the above result still holds true if $u$ is not smooth in which case all the above derivatives are interpreted in a distributional setting. In particular, if $u=\sigma(v)$ where $v$ is smooth and $\sigma$ is our non-differentiable saturation function then the above result can still be applied.

We obtain the following corollary:

Corollary A.2: Consider the system:

$$
\left\{\begin{array}{l}
\dot{x}=A x+B v, \\
y=C x+D v
\end{array}\right.
$$

with $x(0)=0$.

$v=0$ is the only bounded input for which $y(t) \rightarrow 0$ if and only if $(A, B, C, D)$ is left-invertible and has no invariant zeros on the imaginary axis and $D$ is injective.

Moreover, $v=0$ is the only bounded input which has a bounded derivative and for which $y(t) \rightarrow 0$ if and only if $(A, B, C, D)$ is left-invertible and has no invariant zeros on the imaginary axis.

Proof: We apply theorem A.1 with:

$$
\begin{aligned}
R(s) & :=\left[\begin{array}{ll}
s I-A & B
\end{array}\right] \\
S(s) & :=\left[\begin{array}{ll}
C & D
\end{array}\right] \\
V(s) & :=\left[\begin{array}{ll}
0 & I
\end{array}\right] \\
W(s) & :=\left[\begin{array}{ll}
0 & I
\end{array}\right]
\end{aligned}
$$

The extra condition that $D$ must be injective or the derivative of $v$ bounded is really needed. A simple example is $v(t)=\cos \left(t^{2}\right)$ for which the output goes to zero as long as $D=0$. Basically $v$ must either go to zero or start oscillating more and more rapidly. To formalize this concept we define the following class of inputs:

Definition A.3: We define $\mathcal{P}$ as the set of bounded vector-valued function $v$ for which there exists a component $v_{i}$ of $v$ for which there exists $\varepsilon>0, \delta>0$ and a sequence $\left\{t_{n}\right\}\left(t_{n} \rightarrow \infty\right.$ as $n \rightarrow \infty$ ) such that for all $n$ :

for all $t \in\left[t_{n}, t_{n}+\varepsilon\right]: \quad v_{i}(t)>\delta$

or

for all $t \in\left[t_{n}, t_{n}+\varepsilon\right]: \quad v_{i}(t)<-\delta$ 
Our basic claim is that if $v \in \mathcal{P}$ then it can never result in an output which converges to zero as long as the system is left-invertible and has no invariant zeros on the imaginary axis. This is formalized in the following theorem:

Theorem A.4: Assume the system (A.2) is given where $(A, B, C, D)$ is left-invertible and has no invariant zeros on the imaginary axis. Moreover, assume $v$ is bounded and such that $y(t) \rightarrow 0$ as $t \rightarrow \infty$. Finally, assume zero initial conditions. In that case $v \notin \mathcal{P}$.

Remark: The above theorem basically states that either the amplitude of $v$ gets smaller as $t \rightarrow \infty$ or the signal starts oscillating very rapidly as $t \rightarrow \infty$. Our previous example $v(t)=\cos \left(t^{2}\right)$ clearly presents the latter behavior. We will use this result by showing that the difference between two signals achieving regulation will never be in $\mathcal{P}$. But then we can show that any nonlinear feedback can never have asymptotically smaller input then the linear feedback provided both achieve regulation. Hence we can prove that if we can achieve regulation via linear feedback then we can also achieve regulation via linear feedback.

Proof : We prove this theorem by contradiction. Therefore assume that $\varepsilon, \delta$ and $\left\{t_{n}\right\}$ exists satisfying the conditions of the theorem.

Let the transfer matrix of (A.2) be given by $G$. We can factorize $G=\bar{G} H$ where $\bar{G}$ is leftinvertible, has no zeros on the imaginary axis and its direct feedthrough matrix is injective. Moreover, $H$ is square, invertible and has no invariant zeros. The construction of $\bar{G}$ and $H$ can be based on Silverman's algorithm (see [13]). Suppose $w \in \mathbb{R}^{m}$ is such that $G w$ is strictly proper. Then choose $k$ such that $(s+\alpha)^{k} G w$ (with $\alpha>0$ ) is proper but not strictly proper. Let $\Pi$ be the projection onto $w$. Define $G_{1}=G(I-\Pi)+G \Pi(s+\alpha)^{k}$ and $H_{1}=(I-\Pi)+(s+\alpha)^{-k} \Pi$ for which we have $G=G_{1} H_{1}$. If the direct feedthrough matrix of $G_{1}$ is not yet injective we repeat this procedure on $G_{1}$ obtaining $G_{2}$ and $H_{2}$. We can then repeat the procedure on $G_{2}$. After at most $m$ steps (say $l$ steps) this procedure stops and we finally set $\bar{G}=G_{l}$ and $H=H_{l} H_{l-1} \cdots H_{1}$. It is easy to check that these matrices satisfy all our requirements.

Define $s=H v$. Since $H$ is asymptotically stable and $v$ bounded, we obtain from $s=H v$ that $s$ is bounded. Moreover, we have $y=\bar{G} s$ and due to corollary A.2, we know that $s(t) \rightarrow 0$ as $t \rightarrow \infty$.

Let $[K, L, M, 0]$ be a realization of $H$ and denote the state of $H$ by $p$. Define $p_{n}:=p\left(t_{n}\right)$, and define $v_{n} \in L_{2}[0, \varepsilon]$ by $v_{n}(s):=v\left(t_{n}+s\right)$. For all $s \in[0, \varepsilon]$, we have:

$$
M e^{K s} p_{n}+\int_{0}^{s} M e^{K(s-\tau)} L v_{n}(\tau) d \tau=s\left(t_{n}+s\right) \rightarrow 0 \quad \text { as } n \rightarrow \infty
$$

We know $v_{n}$ is bounded and due to the stability of $H$ also $p_{n}$ is bounded. Hence there exists a subsequence $\left\{p_{n_{r}}, v_{n_{r}}\right\}$ such that $p_{n_{r}} \rightarrow p_{*}$ as $r \rightarrow \infty$ and $v_{n_{r}} \stackrel{w}{\rightarrow} v_{*}$. The latter convergence is a convergence in the weak topology and the existence of the subsequence is based on the fact that the unit ball in $L_{2}[0, \varepsilon]$ is weakly compact (see e.g. [12]).

Finally, we note that (A.3) then implies that for all $s \in[0, \varepsilon]$, we have:

$$
M e^{K s} p_{*}+\int_{0}^{s} M e^{K(s-\tau)} L v_{*}(\tau) d \tau=0
$$

Because the system $H$ is invertible and has no zeros, the above implies that $p_{*}=0$ and $v_{*}=0$. 
We complete our proof by showing that $v_{*}=0$ contradicts with the requirements on $v$ outlined in the theorem. After all the conditions in the theorem imply:

$$
\left|\int_{0}^{\varepsilon} v_{n}(s) d s\right|>\varepsilon \delta
$$

for all $n$. But this implies:

$$
\left|\int_{0}^{\varepsilon} v_{*}(s) d s\right|>\varepsilon \delta
$$

which obviously contradicts with $v_{*}=0$. Hence, our initial assumption that $\varepsilon, \delta$ and $\left\{t_{n}\right\}$ exists satisfying the conditions of the theorem was incorrect and our proof is completed.

Note that $v(t)=\cos \left(t^{2}\right)$ would indeed result in $v_{n}$ which converges to zero in the weak topology.

\section{References}

[1] B.D.O. Anderson and J.B. Moore, Optimal control: linear quadratic methods, Prentice-Hall, Englewood Cliffs, 1989.

[2] J.J.A.M. Brands, M.L.J. Hautus, "Asymptotic properties of matrix differential operators", J. of Math. Anal. \& Appl. Vol. 87, No. 1, pp. 199-218, 1982.

[3] C.-T. Chen, Linear System Theory and Design, Holt, Rinehart and Winston, New York, 1984.

[4] B.A. Francis, "The linear multivariable regulator problem", SIAM J. Contr. \& Opt., Vol. 15, No. 3, pp. 486-505, 1977.

[5] A.T. Fuller, "Linear control of non-linear systems", Int. J. Control, Vol. 5, No. 3, pp. 197-243, 1967.

[6] A.T. Fuller, "In-the-large stability of relay and saturating control systems with linear controller", Int. J. Control, Vol. 10, No. 4, pp. 457-480, 1969.

[7] M. Hautus, "Linear matrix equations with applications to the regulator problem," in Outils and Modèles Mathématique pour l'Automatique, I.D. Landou, Ed. Paris: C.N.R.S., pp. 399-412, 1983.

[8] Z. Lin and A. Saberi, "Semi-global exponential stabilization of linear systems subject to 'input saturation' via linear feedbacks", Systems \& Control Letters, vol. 21, no. 3, pp. 225-239, 1993.

[9] Z. Lin and A. Saberi, "Semi-global exponential stabilization of linear discrete-time systems subject to 'input saturation' via linear feedbacks", to appear in Systems $\&$ Control Letters.

[10] Z. Lin and A. Saberi, "A low-and-high gain approach to semi-global stabilization and/or semi-global practical stabilization of a class of linear systems subject to input saturation via linear state and output feedback," Proc. 32nd IEEE Conference on Decision and Control, 1993.

[11] Z. Lin and A. Saberi, "A semi-global low-and-high gain design technique for linear systems with input saturation - stabilization and disturbance rejection," submitted to International Journal of Robust and Nonlinear Control, 1993.

[12] W. Rudin, Real and complex analysis, Third Ed., McGraw-Hill, 1987.

[13] L.M. Silverman and H.J. Payne, "Input-output structure of linear systems with application to the decoupling problem", Siam J. Contr., Vol. 9, No. 2, pp. 199-233, 1971. 
[14] E.D. Sontag and H.J. Sussmann, "Nonlinear output feedback design for linear systems with saturating controls," Proc. 29th IEEE Conf. Decision and Control, pp. 3414-3416, 1990.

[15] H.J. Sussmann and Y. Yang, "On the stabilizability of multiple integrators by means of bounded feedback controls", Proc. 30th CDC, Brighton, U.K., pp. 70-72, 1991.

[16] H.J. Sussmann, E.D. Sontag and Y. Yang, "A general result on the stabilization of linear systems using bounded controls", Preprint, 1993.

[17] A.R. Teel, "Global stabilization and restricted tracking for multiple integrators with bounded controls", Systems \& Control Letters, Vol. 18, No.3, pp. 165-171, 1992.

[18] A.R. Teel, Feedback Stabilization: Nonlinear Solutions to Inherently Nonlinear Problems, Ph.D dissertation, College of Engineering, University of California, Berkeley, CA, 1992.

[19] H.L. Trentelman, "Families of linear-quadratic problems: continuity properties", IEEE Trans. Aut. Control, Vol. 32, pp. 323-329, 1987.

[20] J.C. Willems, "Least squares stationary optimal control and the algebraic Riccati equation", IEEE Trans. Aut. Control, Vol. 16, pp. 621-634, 1971.

[21] J.C. Willems, A. Kitapçi, L.M. Silverman, "Singular optimal control: a geometric approach", SIAM J. Contr. Opt., Vol. 24, pp. 323-337, 1986.

[22] W.M. Wonham, Linear Multivariable Control: A Geometric Approach, 2nd ed. New York: Springer-Verlag, 1979. 